4-1-1999

\title{
On the Detachment of Step-Tapered Doublers: Part 2-Evolution of Pressure Loaded Structures
}

Anette M. Karlsson

Cleveland State University, a.karlsson@csuohio.edu

W. J. Bottega

Rutgers University - New Brunswick/Piscataway

Follow this and additional works at: https://engagedscholarship.csuohio.edu/enme_facpub

Part of the Mechanical Engineering Commons

How does access to this work benefit you? Let us know!

Publisher's Statement

NOTICE: this is the author's version of a work that was accepted for publication in International Journal of Solids and Structures. Changes resulting from the publishing process, such as peer review, editing, corrections, structural formatting, and other quality control mechanisms may not be reflected in this document. Changes may have been made to this work since it was submitted for publication. A definitive version was subsequently published in International Journal of Solids and Structures, 36, 11, April 1, 1999; 10.1016/S0020-7683(98)00053-5

\section{Original Citation}

Karlsson, A. M., and Bottega, W. J., 1999, "On the Detachment of Step-Tapered Doublers: Part 2-evolution of Pressure Loaded Structures," International Journal of Solids and Structures, 36(11) pp. 1625-1651.

This Article is brought to you for free and open access by the Mechanical Engineering Department at EngagedScholarship@CSU. It has been accepted for inclusion in Mechanical Engineering Faculty Publications by an authorized administrator of EngagedScholarship@CSU. For more information, please contact library.es@csuohio.edu. 


\title{
On the detachment of step-tapered doublers: Part 2 - evolution of pressure loaded structures
}

\author{
A. M. Karlsson, W. J. Bottega* \\ Department of Mechanical and Aerospace Engineering, Rutgers University, Piscataway, NJ 08854-8058, U.S.A.
}

\section{Introduction}

Configurations corresponding to the adherance of a secondary structure to a primary structure forming a 'composite structure' arise in a variety of settings, an example of which is that of a patch or 'doubler' adhered to a thin base structure such as a plate or shell. Debonding of such configurations is an important issue as such behavior will evidently compromise the effectiveness and integrity of structures of this class. Prior work pertaining to various issues with regard to the debonding of uniform doublers was reported by Bottega (1995), Bottega and Loia (1996), Bottega and Loia (1997) and Loia and Bottega (1995). Both flat and cylindrical structures were studied for various loading and support conditions. As many preliminary designs and tests employ a layerwise step-taper of the patch at its edges, in order to counter effects associated with sudden

\footnotetext{
*Corresponding author. Fax: 001732445 5313; e-mail: bottega@rci.rutgers.edu
} 
changes in geometry, (see, for example, Raizenne et al., 1995) it is of interest to examine how such tapering affects debonding of the patch from the base structure.

In Part 1 of the present study (Bottega and Karlsson, 1998) the formulation for both plate and shell structures with layered patches was presented simultaneously, where layerwise tapering of the edges of the patch was incorporated. The problems were approached as propagating boundary problems, incorporating an appropriate thin structure theory for each of the individual layers of the patch and for the base structure. The composite patch was modeled as an assemblage of its constituent layers, and the 'composite structure' as an assemblage of the composite patch and base structure. The effects of a contact zone adjacent to the bond zone boundary, and also the presence of edge-point contact alone, as well as the situation of no contact of the debonded segments of the composite structure were included in the formulation. The self-consistent governing equations, boundary and matching conditions, and tranversality conditions which establish the locations of the propagating bond zone and contact zone boundaries associated with equilibrium configurations of the evolving composite structure were established. The former tranversality conditions yielded the self-consistent and physically interpretable expressions for the energy release rates which govern debonding. Conditions pertaining to edge-point contact were likewise established. Results of numerical simulations, based on analytical solutions, corresponding to applied in-plane tension and to three-point transverse loading of flat plate configurations were also presented. In the present study we apply the formulation presented in Part 1 to study the intricate and complex debonding behavior of pressure loaded structures, a loading of great practical significance. Both flat and curved configurations are studied for several support conditions. It will be seen that the degree of taper often influences the debonding behavior in undesirable ways, and that such modifications of the patch should be carefully considered.

\section{Problem description}

We first present a brief description of the structures to be considered. Both flat and curved structures are considered. In keeping with earlier work (Bottega, 1995; Bottega and Loia, 1996; Bottega and Loia, 1997), all length scales are normalized with respect to the dimensional radius $\bar{R}$ of the base panel for the case of cylindrical (shell) structures, and with respect to the dimensional half-length $\bar{L}_{0}$ of the span of the base panel for the case of flat (plate) structures.

Let us consider a base structure of normalized half-length $L_{0}$ to which a layered doubler (patch) of half-length $L_{1} \leqslant L_{0}$ is adhered over the center of the span as shown in Fig. 1. Let the patch be bonded to the base panel over the region $s \in\left[0, a \leqslant L_{1}\right]$ where $s$ is the normalized coordinate paralleling the upper surface of the base structure and originating at the center of the span as indicated. At this point let us introduce the conjugate bond zone size $a^{*}=L_{0}-a$, which locates the bond zone boundary from the edge of the base structure, as shown in the figure. In addition, we consider a region of sliding contact (the contact zone) adjacent to the bond zone within the region $s \in\left[a, b \leqslant L_{1}\right]$. It was shown in Part 1 (Bottega and Karlsson, 1998) that, for the class of problems under consideration, either $b=a$ (no contact) or $b=L_{1}$ (full contact). An intermediate or propagating contact zone boundary $b$ was shown not be an issue. We also consider the situation of edge-point contact, where the patch edge maintains sliding contact with the base structure at $s=L_{1}$ but is lifted away at all interior points $s \in\left(a, L_{1}\right)$ within the debonded region. 


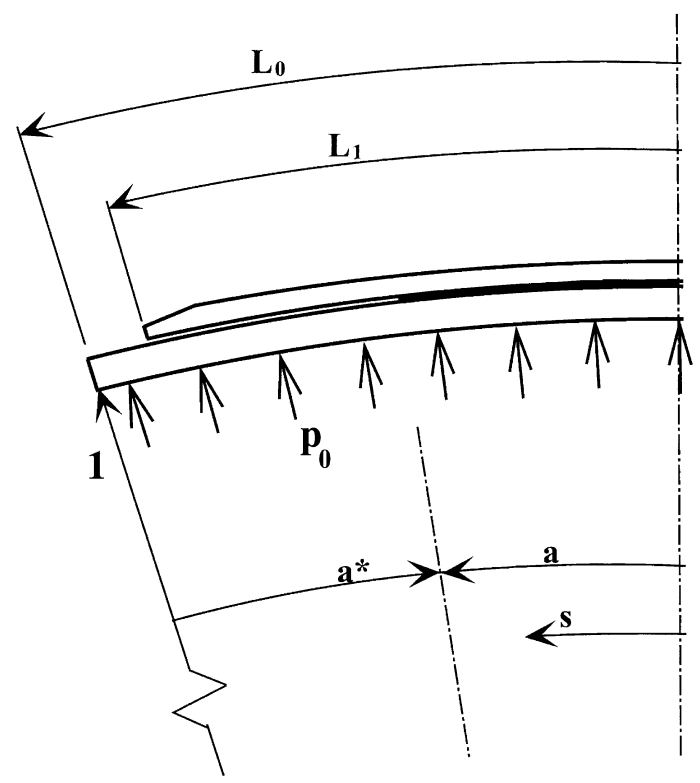

Fig. 1. Patched panel subjected to applied pressure. (Shown for cylindrical structure.)

The structure is considered to be subjected to an applied transverse pressure of normalized intensity $p_{0}$ as indicated. In addition, the strength of the bond at the interface between the first layer of the patch $(\alpha=1)$ and the base panel is characterized by the normalized energy per unit area $\gamma$. We also introduce the renormed pressure $p^{*}$. These parameters are related to their dimensional counterparts, $\bar{p}$ and $\bar{\gamma}$, as

$$
\begin{aligned}
& p_{0}=\bar{p} \bar{L}^{3} / \overline{D_{0}}, \quad p^{*}=p_{0} / \sqrt{2 \gamma} \\
& \text { and } \gamma=\bar{\gamma} \bar{L}^{2} / \bar{D}_{0}
\end{aligned}
$$

where $\quad \bar{L}=\bar{R} \quad$ (for cylindrical structures) and $\bar{L}=\bar{L}_{0}$ (for flat structures).

In eqns (1a) and (2), the parameter $\overline{D_{0}}$ corresponds to the dimensional bending stiffness of the base panel. For consistency we introduce, at this point, the renormed centerspan deflection $\Delta_{0}^{*}$ and normalized global stiffness $K^{*}$ where

$$
\Delta_{0}^{*}=\Delta_{0} / \sqrt{2 \gamma}, \quad K^{*}=p^{*} / \Delta_{0}^{*}
$$

and $\Delta_{0}$ corresponds to the normalized (transverse) centerspan deflection [i.e. the ratio of the dimensional deflection to the appropriate length scale $\bar{L}$ as defined in eqns (3a,b)].

The patches under consideration are taken to be comprised of $n$ elastic layers, as shown in Fig. 2 , with the normalized modulus ratio of layer $\alpha, E_{\alpha}$, being given by

$$
E_{\alpha}=\bar{E}_{\alpha} / \bar{E}_{0} \quad \text { or } \quad E_{\alpha}=\frac{\bar{E}_{\alpha} /\left(1-v_{\alpha}^{2}\right)}{\bar{E}_{0} /\left(1-v_{0}^{2}\right)} . \quad(\alpha=1,2, \ldots, n)
$$




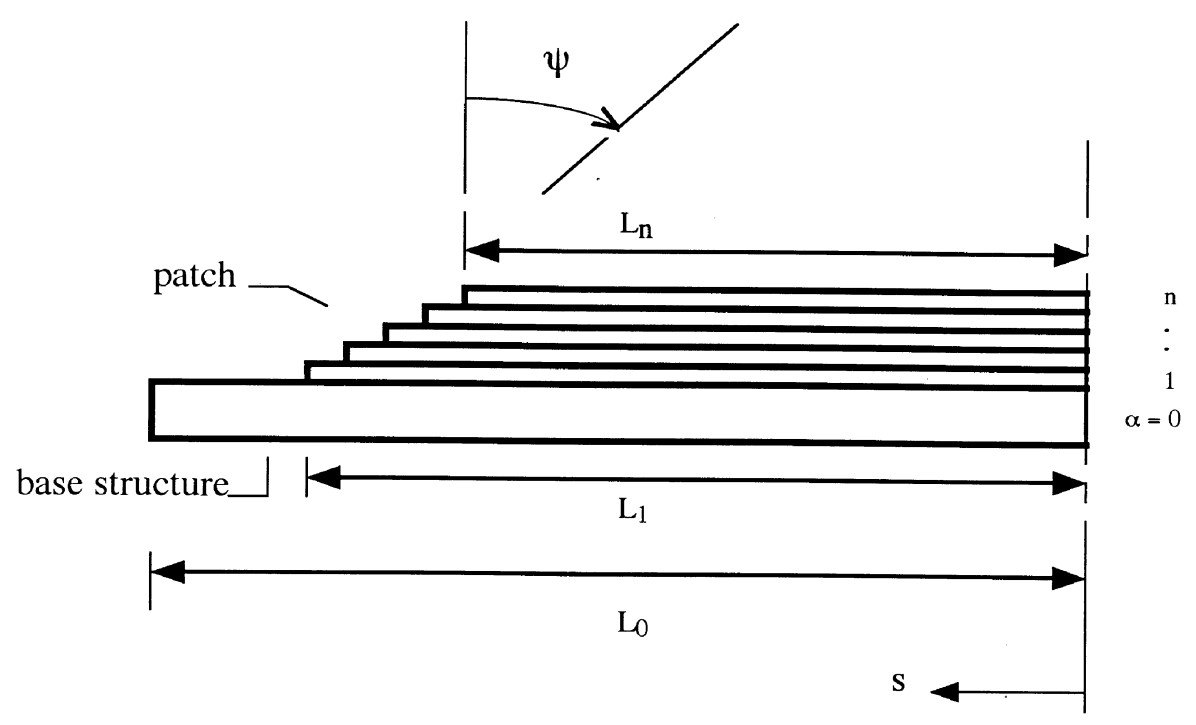

Fig. 2. Geometry of step-tapered patch with base panel. (Shown for flat panel.)

In eqns (5), $\bar{E}_{0}$ and $\bar{E}_{\alpha}$ correspond to the dimensional elastic moduli of the base panel and layer $\alpha$ of the patch, respectively, and $v_{0}$ and $v_{\alpha}$ represent the associated Poisson's ratios. The edge of the patch is considered to be tapered step-wise, as indicated, with the 'taper angle' $\psi: 0 \leqslant \psi<\pi / 2$, as defined in Fig. 2, characterizing the degree of taper. We note that the value $\psi=0$ corresponds to the untapered case.

The analysis in each case will be conducted based on the analytical solutions to the appropriate linearized versions of the formulation presented in Part 1 of this study (Bottega and Karlsson, 1998). It was shown therein that for the case of pinned supports (free or fixed with regard to circumferential/in-plane translation) a contact zone would not occur. Similar arguments prohibit the occurence of edge-point contact as well, for this type of support. Thus, only configurations corresponding to no contact of the delaminated segments of the patch and base structure are physically realizable for the case of pinned supports. For the case of clamped support conditions ( free or fixed with regard to translation), however, full contact, edge-point contact and no contact configurations may all be physically realizable. In the event that more than one configuration is admissible for a given base structure, patch and bond zone size, the 'preferred' configuration will be taken to be that which corresponds to the lowest total energy of the system. In this regard, the total energy of the system will be characterized by the normalized work per unit pressure

$$
\left.\mathscr{W}^{*} \equiv \mathscr{W}\right|_{p 0=1}
$$

where the explicit form of the normalized work $\mathscr{W}$ is given by eqn $(9 \mathrm{c})$ of Part 1 of this study (Bottega and Karlson, 1998).

In the following sections, results are presented for both flat and curved structures subjected to pressure loading. For the purposes of comparison with prior results pertaining to untapered patches we consider, in each case, specific structures where the patch is comprised of four layers 
$(n=4)$ having identical material properties $E_{\alpha}=E_{\mathrm{p}}(\alpha=1-4)$ and normalized thicknesses $h_{\alpha}=h_{\mathrm{p}} / n=h_{0} / n$, where $h_{0}$ is the thickness of the base structure and $h_{\mathrm{p}}$ is the total thickness of the patch in the untapered region. Since, in prior studies (Bottega, 1995; Bottega and Loia, 1996; Bottega and Loia, 1997) relatively long patches were generally seen to yield the most desirable behavior with regard to the debonding of untapered patches, the total length of the patch for the present structure is taken as the same as that of the base panel $\left(L_{1}=L_{0}\right)$. For similar reasons, we consider the layers of the patch with intermediate modulus ratio $E_{\mathrm{p}}=1$ throughout. For the purposes of comparison with prior results pertaining to untapered patches mentioned earlier, the thickness to length ratios of the base structures under consideration are taken as 0.05 throughout. (Given the normalization described by eqns (3a) and (3b), such a ratio translates to a base structure of normalized thickness $h_{0}=0.02$ and half-length $L_{0}=0.4$ for the curved structures under consideration, while $h_{0}=0.05$ and $L_{0}=1$ for the flat structures.) In all cases the taper angle $\psi$ is varied over a wide range of values. In this way, an evaluation of the effects of edge taper can be made. We first present and discuss results pertaining to flat structures.

\section{Evolution of flat structures}

In this section, results are presented for the case of patched plate structures. We consider the two opposite extremes of support conditions. These correspond to the situations where the edges of the base structure are: (i) hinged-so as to allow rotation and free-so as to allow in-plane translation (hinged-free), and (ii) clamped - so as to prohibit rotation and fixed - so as to prohibit in-plane translation (clamped-fixed). We consider the less constrained system first.

\subsection{Hinged-free supports}

We first consider a flat structure where the edges of the base plate are free to rotate and to translate axially. It was shown in Part 1 (Bottega and Karlsson, 1998) that for the case of a flat structure with hinged supports ( free or fixed), the debonded region will not possess a contact zone. Similar arguments indicate that such conditions will not admit edge contact either. Hence, for hinged support conditions, we only consider propagation of the debonded region without contact, i.e. with the entire debonded segment of the patch lifted away from the base plate. For this case, we present results pertaining to taper angles, $\psi$, in the range $75^{\circ} \leqslant \psi \leqslant 85^{\circ}$ where $\psi=85^{\circ}$ is the most severe tapering (see Fig. 2). Results pertaining to lower taper angles show similar trends and characteristics and are thus omitted for brevity.

The threshold curves expressed in terms of the renormed pressure $p^{*}$ as a function of the conjugate bond zone size $a^{*}$, and the renormed characteristic displacement $\Delta_{0}^{*}$ as a function of $a^{*}$ for the hinged-free plate with taper angles $\psi=75,80$ and $85^{\circ}$ are displayed in Figs $3 \mathrm{a}$ and $3 \mathrm{~b}$, respectively. The associated stiffness degradation curves are displayed in Fig. 3c. The corresponding results for an untapered patch $\left(\psi=0^{\circ}\right)$ are also displayed for comparison. The discontinuities in the present set of threshold curves arise from the discontinuities (steps) in the structure at the points where a new layer comes into effect as the conjugate bond zone size is varied. (The paths 

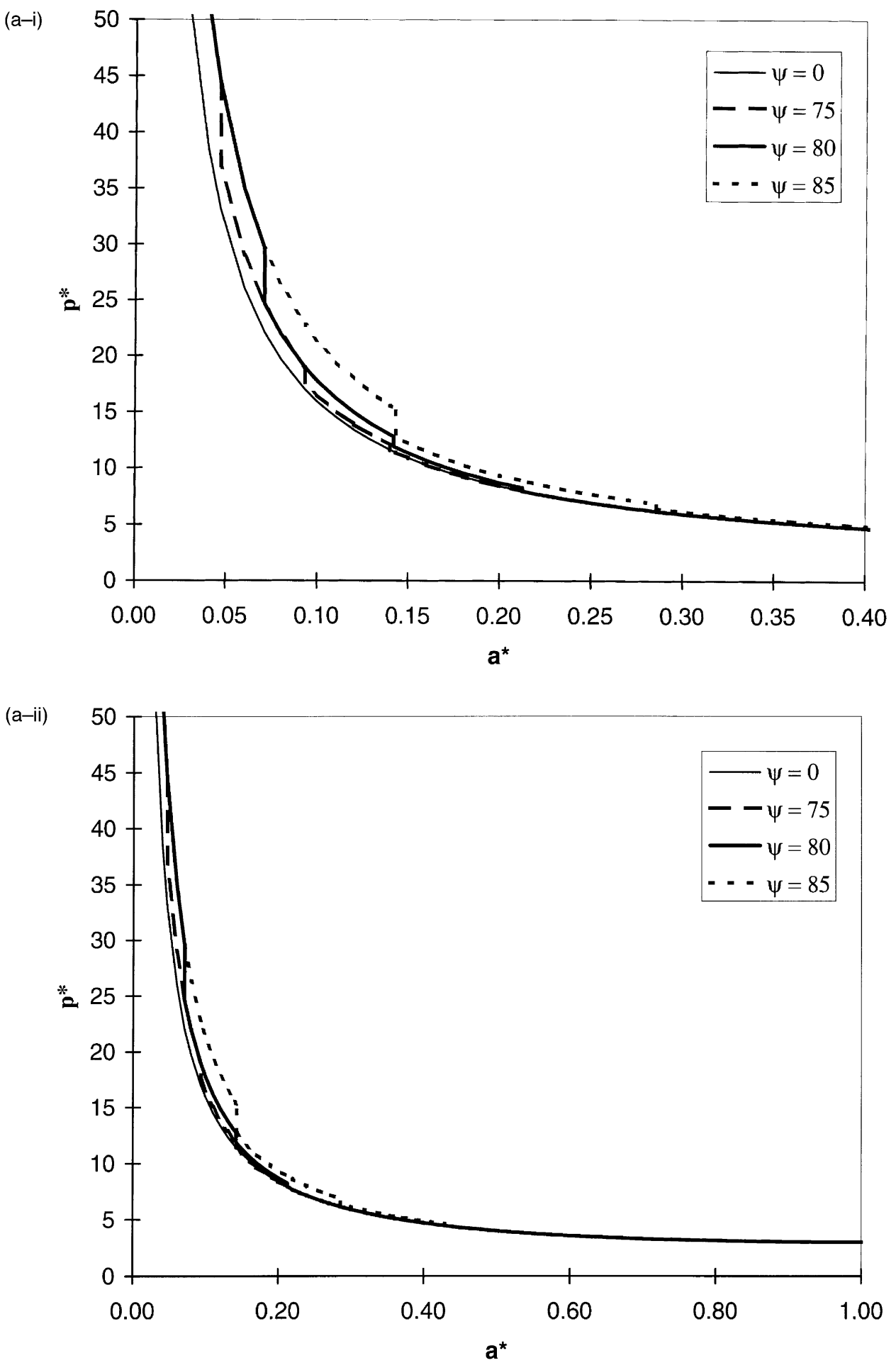

Fig. 3. Threshold curves/delamination paths corresponding to patched plates with hinged-free supports, for various taper angles $\left(\psi=0,75,80\right.$ and $\left.85^{\circ}\right)$ : (a-i,ii) renormed pressure vs conjugate bond zone size; (b) renormed centerspan deflection vs conjugate bond zone size; (c) stiffness degradation curves. 
(b)

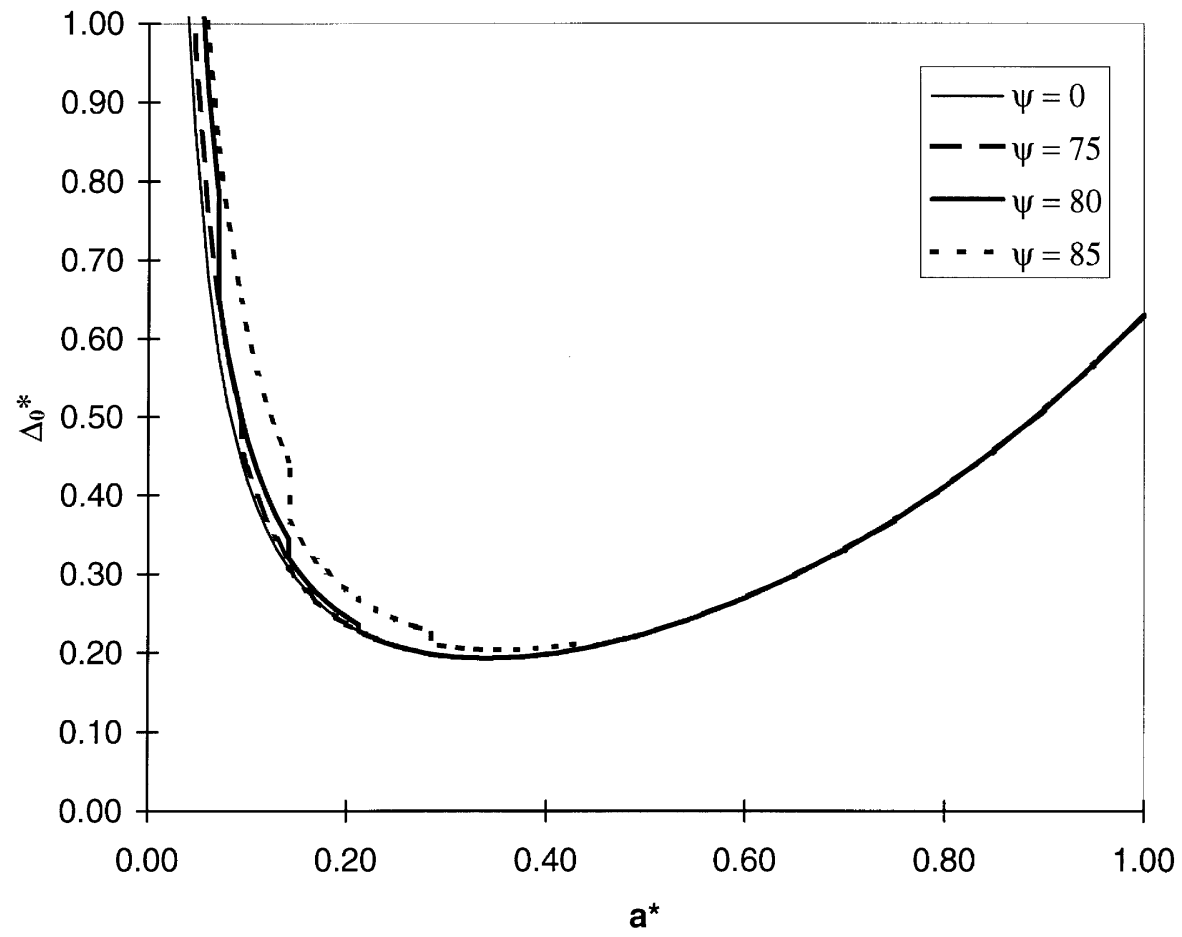

(c)

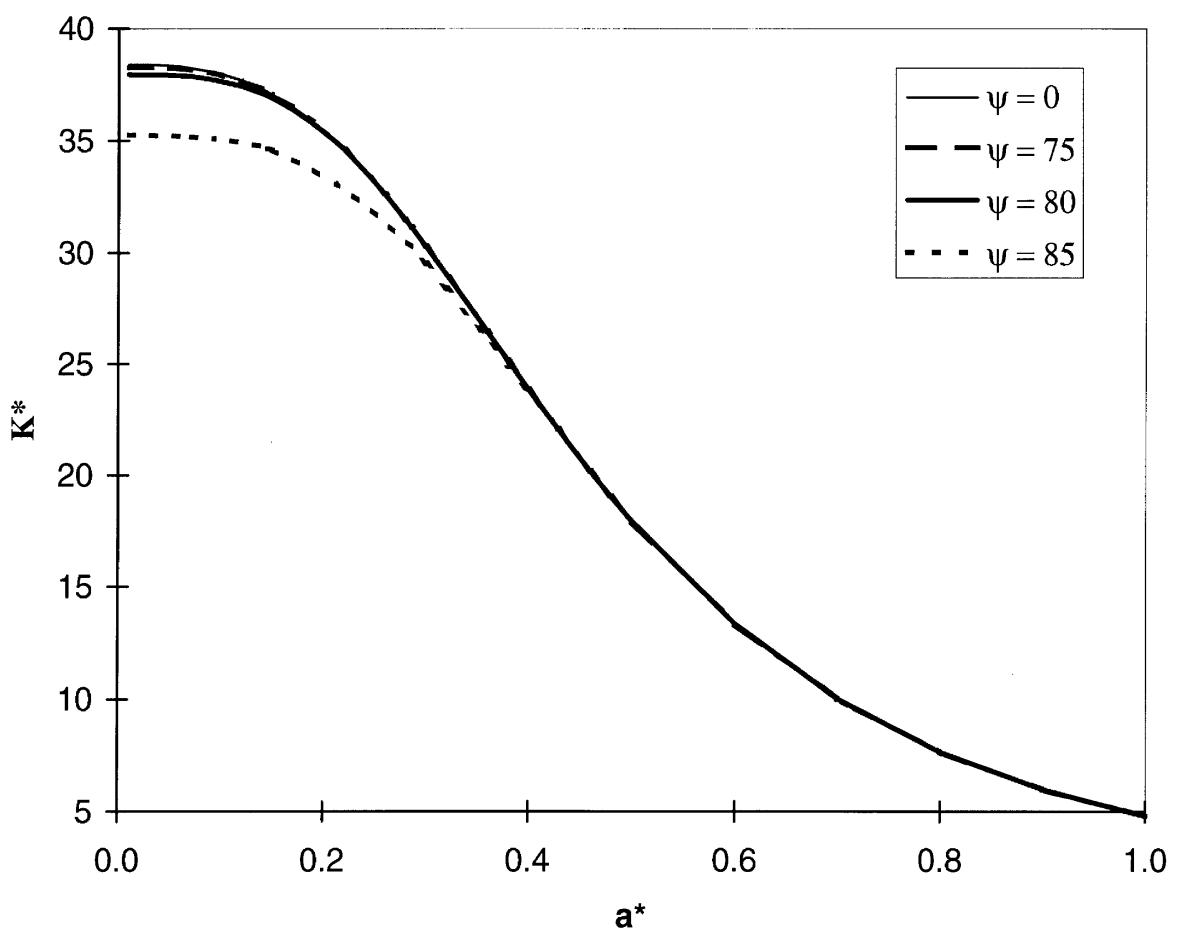

Fig. 3-continued. 
displayed in Fig. 3a-i focus on the range $0 \leqslant a^{*} \leqslant 0.4$ in order to magnify the differences associated with varying the taper angle. The corresponding paths are shown for the full range of values of $a^{*}$ in Fig. 3a-ii.) All of the equilibrium paths approach their counterparts for the untapered case (Bottega, 1995), for large enough $a^{*}$, and become identical to those paths once the last step of the patch is achieved for any given $\psi$. The curves in Fig. 3a show $p^{*}$ monotonically decreasing with $a^{*}$ throughout the length of the structure, while those displayed in Fig. $3 \mathrm{~b}$ possess a relative minimum around $a^{*} \approx .34$ with $\Delta_{0}^{*}$ monotonically increasing with $a^{*}$ away from this point. Upon examination of Fig. 3a, it may be seen that tapering of the edge of the patch increases the critical pressure above that for the untapered case in correlation with the degree of taper, and has the largest effect when $a^{*}$ is located within the region where only one of the patch's four layers is present (i.e., the region of lowest stiffness), with the effect diminishing accordingly. It may be seen from the figure that, for pressure controlled loading, debonding will propagate in an unstable and catastrophic manner for any taper angle once the critical level of pressure is reached for any given initial $a^{*}=a_{0}^{*}$.

Upon examination of Fig. 3b, it may be seen that tapering the edge of the patch increases the critical deflection in the range of values of $a^{*}$ where tapering occurs, for deflection controlled loading, in the same manner as for the case of force (pressure) controlled loading. For deflection controlled loading, however, debonding is seen to occur in an unstable and catastrophic manner, in an unstable followed by a stable manner, or in a stable manner, once the critical deflection level is reached depending on the initial conjugate bond zone size $a^{*}=a_{0}^{*}$, for any degree of tapering. It is important to note that though the critical deflection is seen to increase when edge tapering is introduced, the extent of unstable growth is seen to be increased beyond that for the untapered case for situations where the initial conjugate bond zone size is such that debonding would initially propagate in an unstable manner. For certain initial conjugate bond zone sizes, catastrophic debonding is indicated where bounded unstable growth of the debonded region followed by stabilization of the growth process was indicated for the untapered case. Thus, the benefit of the increase in threshold level of the characteristic deflection may be offset by the detriment of decreasing the stability of the debonding process for deflection controlled loading situations.

\subsection{Clamped-fixed supports}

We next consider the evolution of a patched plate when the edges of the base structure are clamped - so as to prohibit rotation and fixed - so as to prohibit in-plane translation. It was shown in Part 1 (Bottega and Karlsson, 1998) that for the case of clamped support conditions (fixed or free), a full contact zone, edge point contact, or no contact of the delaminated segments of the patch and the base plate are all possible configurations. In the following, we first discuss the hierarchy among admissible configurations. We then proceed to study how the degree of taper affects the debonding behavior. In this regard, results are presented for taper angles, $\psi$, in the range $30^{\circ} \leqslant \psi \leqslant 87^{\circ}$.

As discussed in section 2, in instances where all three configurations ( full contact, edge point contact, no contact) are admissible, the 'preferred' configuration will be taken to be the one corresponding to the lowest energy per unit load as characterized by the work per unit pressure. The work per unit pressure, $\mathscr{W}^{*}$, is displayed as a function of the conjugate bond zone size, $a^{*}$, (in Fig. 4a) for the representative taper angle, $\psi=60^{\circ}$. While the no contact configuration admits a possible solution for all values of $a^{*}$, the other two configurations do not. The corresponding 
(a)

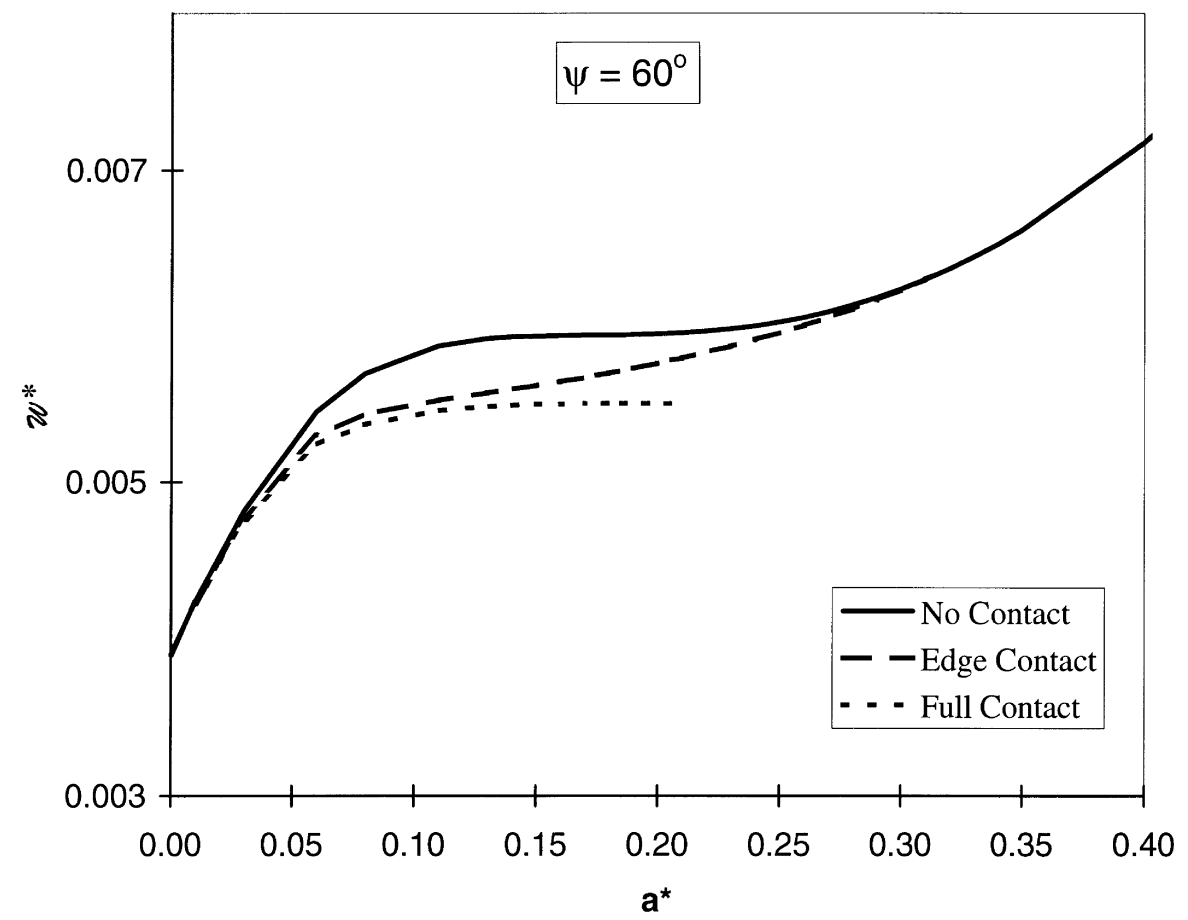

$(\mathrm{b}-\mathrm{i})$

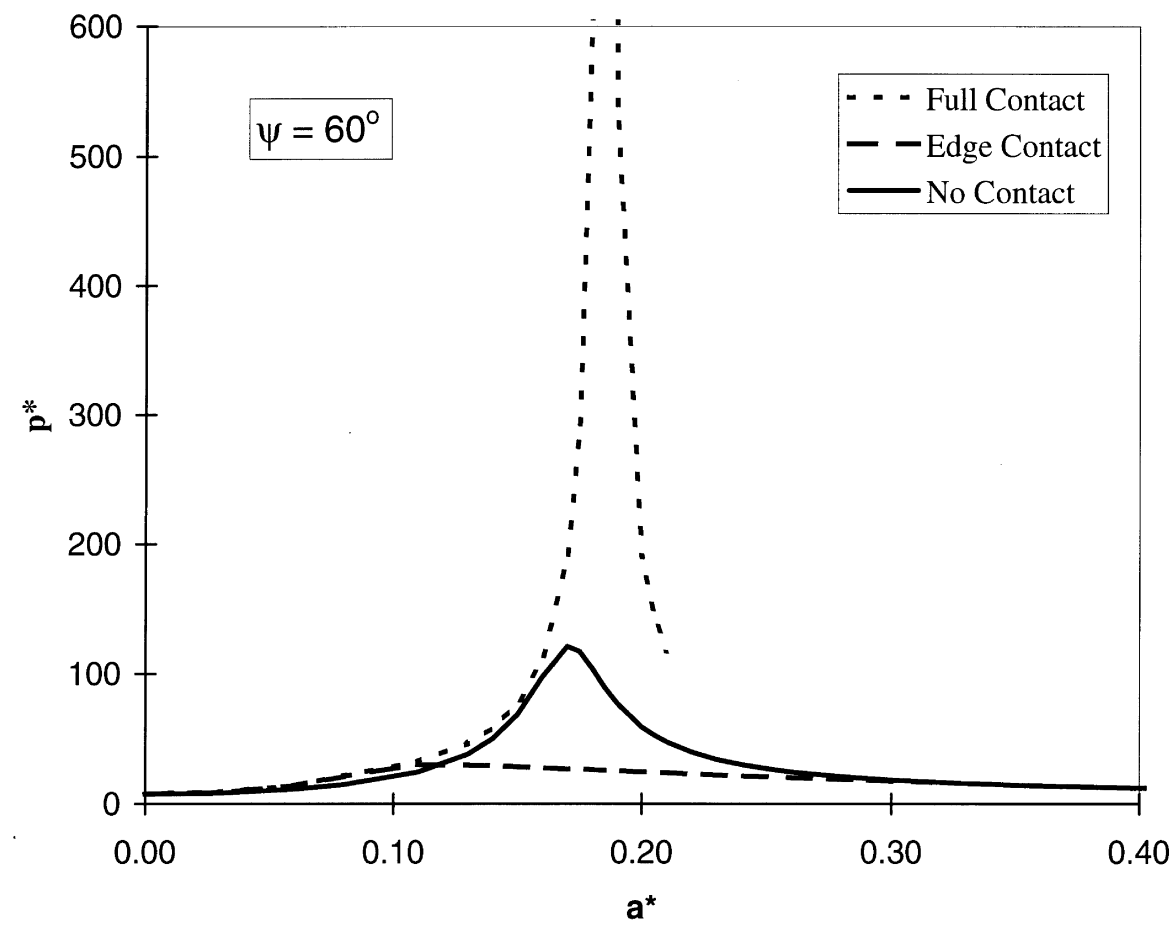

Fig. 4. Comparison of equilibrium paths corresponding to configurations with full contact zone, edge-point contact and no contact for a representative patched plate with clamped-fixed supports, $\psi=60^{\circ}$ : (a) total normed work per unit pressure vs conjugate bond zone size; (b-i,ii) renormed pressure vs conjugate bond zone size; (c) renormed centerspan deflection vs conjugate bond zone size; (d) stiffness degradation curves. 


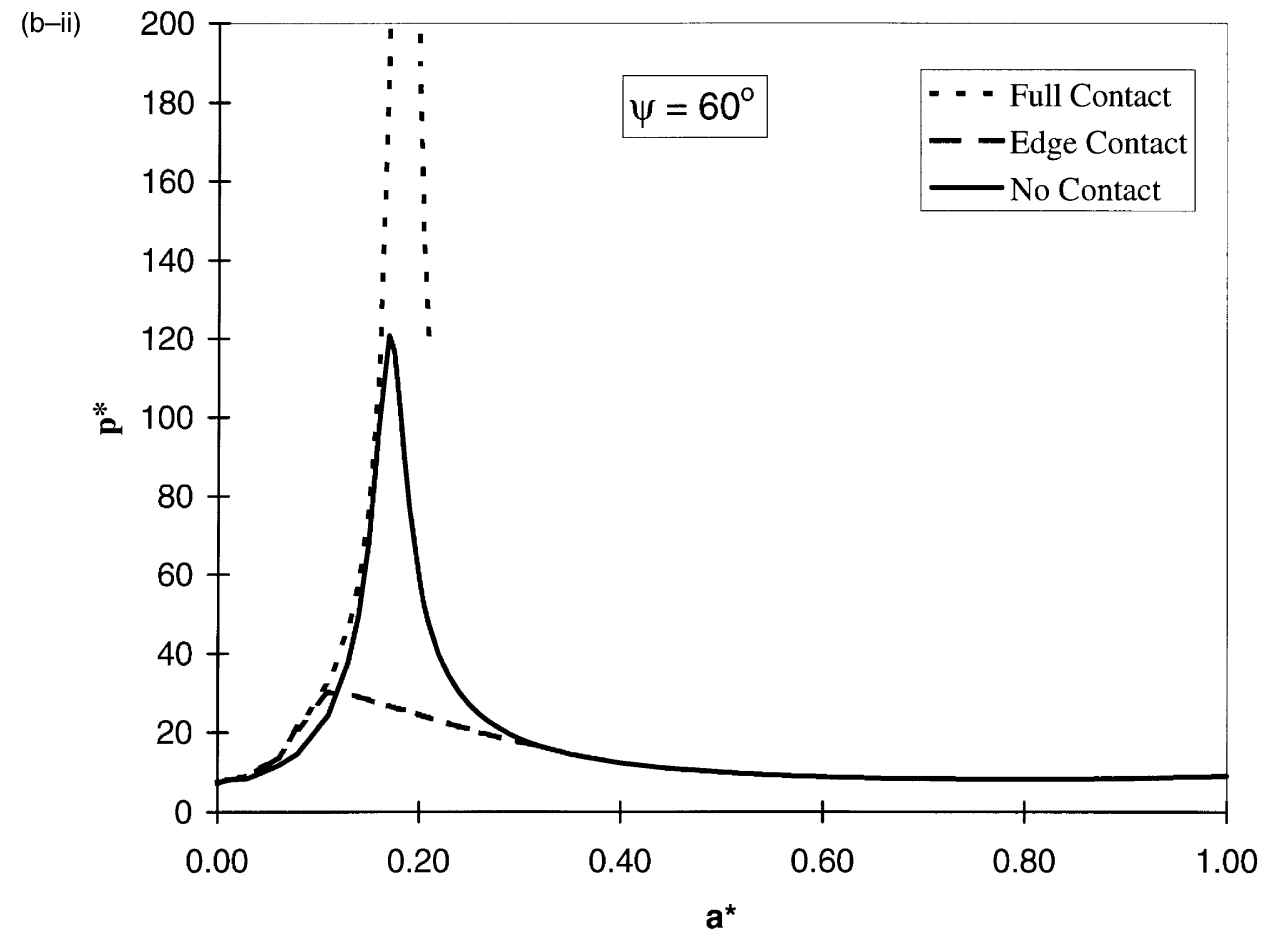

(c)

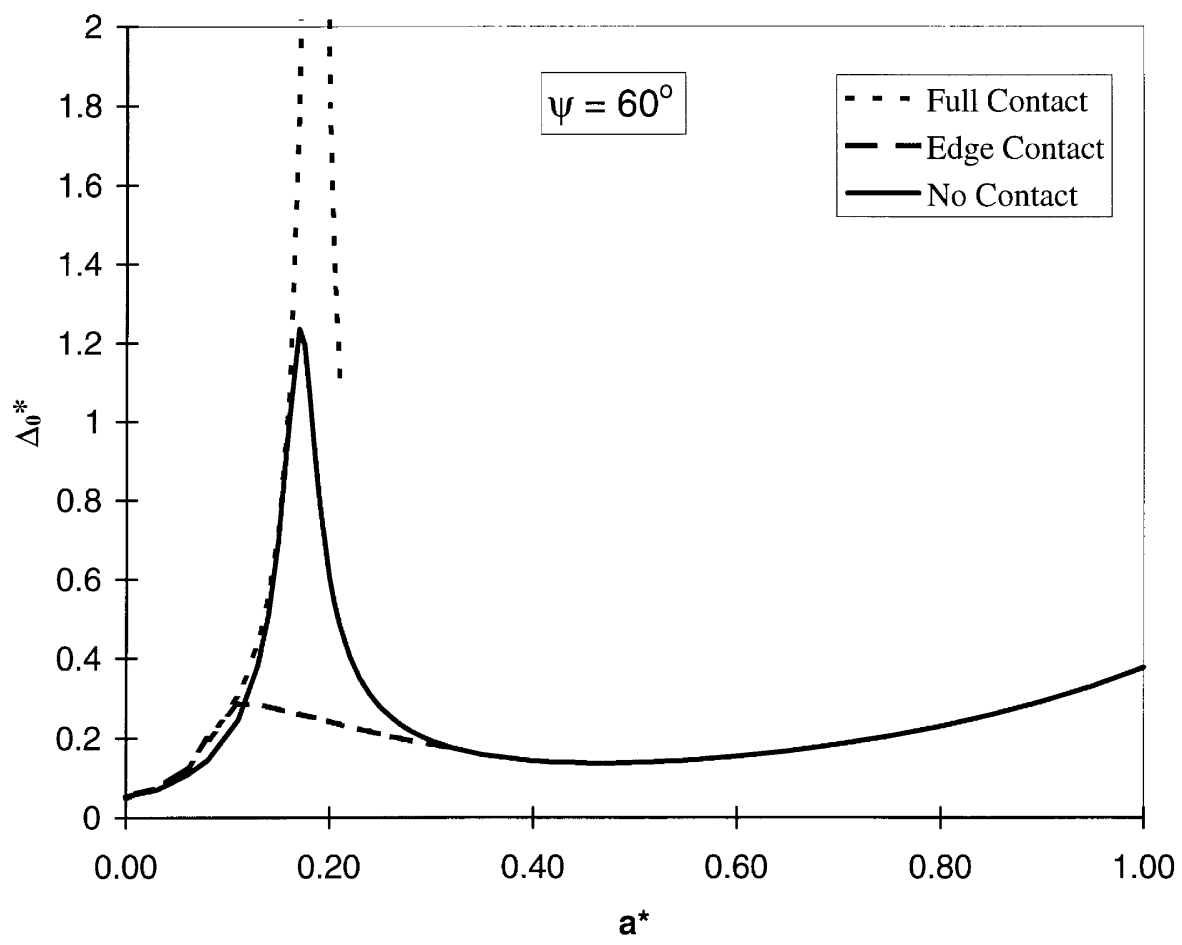

Fig. 4-continued. 


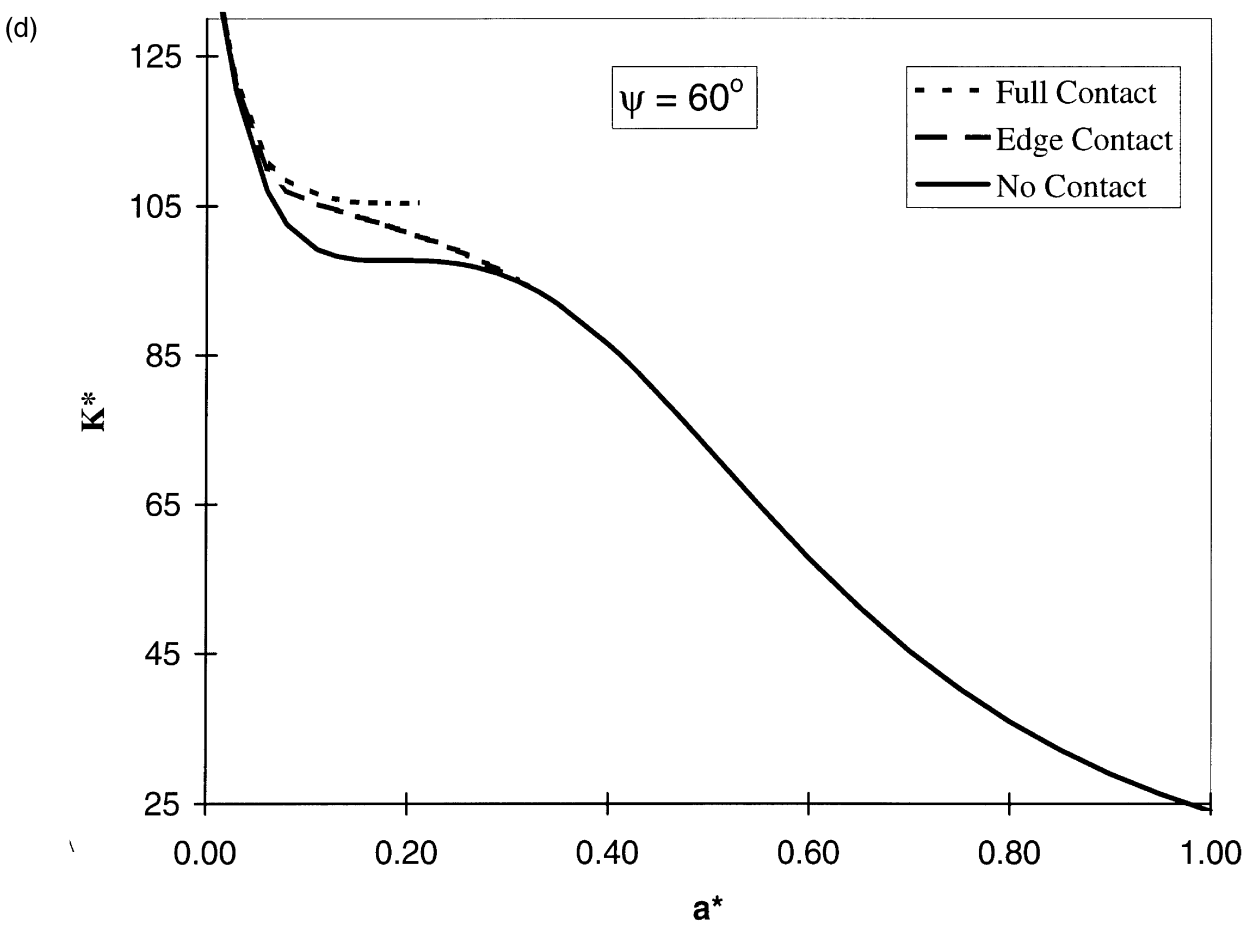

Fig. 4-continued.

curves are 'cut off' outside their admissible range (the range of $a^{*}$ for which admissible solutions are found). $\dagger$ It may be seen from the figure, that when such configurations are possible, the configuration corresponding to a full contact zone is the one of lowest total energy, followed by that corresponding to edge-point contact. Consequently, the configuration corresponding to no contact of the debonded segments is seen to possess the highest total energy and hence is the least preferred configuration. Similar results were obtained for all taper angles considered, but are omitted for brevity. Thus when two or more of the three possible configurations are admissible for a given structure and bond zone size, the hierarchy of 'preference' of the system is found to be: (1) full contact zone; (2) edge-point contact; (3) no contact. The corresponding threshold and stiffness degradation curves $\left(\psi=60^{\circ}\right)$, for each configuration (where admissible) are shown in Figs $4 \mathrm{~b}-\mathrm{d}$, with those expressed in terms of the renormed pressure $p^{*}$ as a function of $a^{*}$, displayed in Figs $4 \mathrm{~b}-\mathrm{i}$,ii. It may be seen in Fig. $4 \mathrm{~b}$ that the threshold curve corresponding to the (preferred) full contact zone configuration possesses an asymptote at $a^{*}=0.186$ which divides the curve into a stable branch to its left $\left(a^{*}<0.186\right)$ and an unstable branch to its right $\left(a^{*}>0.204\right)$. For the case of stable debonding (initial $a^{*}=a_{0}^{*}$ to the left of the asymptote), it may be seen that debonding effectively arrests as $a^{*}$ approaches the asymptote. The configuration corresponding to edge-

$\dagger$ Though difficult to see within the resolution of the figure, the curve corresponding to edge contact cuts off at $a^{*}=0.321$. 
point contact is the preferred configuration for $0.214<a^{*}<0.321$. Thus for situations when the structure is initially debonded such that $0.186<a_{0}^{*}<0.214$ debonding ensues, once the critical pressure level is reached, and propagates in an unstable fashion until $a^{*}=0.214$ at which point the debonded segment of the patch lifts away from the base plate at all interior points but maintains sliding contact at its edge. Debonding continues in an unstable manner, and when $a^{*}=0.321$ the edge of the patch lifts away from the base plate ('lift off') with debonding continuing to propagate in an unstable and catastrophic manner without any contact. For a structure with an initial debonded region such that $0.214<a_{0}^{*}<0.321$ debonding ensues when the critical pressure level indicated by the edge contact path is achieved. (It may be observed that this threshold level is substantially lower than that which would be predicted if edge contact were not taken into account.) Once debonding begins, it is seen to progress in an unstable and catastrophic manner, with complete 'lift off' occuring when $a^{*}=0.321$. Finally, if the initial size of the debonded region is such that $a_{0}^{*}>0.321$, debonding occurs in a catastrophic, unstable followed by stable, or in a (rapidly) stable manner depending on the initial value of the conjugate bond zone size. With the above scenario established, we next investigate the influence of the degree of taper of the patch.

Threshold curves expressed in terms of the renormed pressure $p^{*}$ as a function of the conjugate bond zone size $a^{*}$ are displayed in Fig. 5a for the taper angles $\psi=30,60$, and 70 , and in Fig. 5b for the taper angles $\psi=80,85$, and $87^{\circ}$. The path corresponding to an untapered patch $\left(\psi=0^{\circ}\right)$ is also shown in each figure for comparison. In each case only those paths associated with the 'preferred' configuration within a given range of $a^{*}$ are displayed. The discontinuity in the threshold curve for a given value of $\psi$ corresponds to the transition between equilibrium configurations possessing a full contact zone and those for which there is edge-point contact only. The transition from states for which there is edge-point contact alone to those for which there is no contact of the debonded segments ('lift off') appears smooth. [We note that, for any value of $\psi$, the path associated with no contact approaches the corresponding path for the untapered case (Bottega, 1995), and becomes identical with that path once the last step of the patch is encountered. As such, all paths become identical to the no contact path of Fig. 4b-ii for large enough values of $a^{*}$.] It may be seen, upon consideration of Fig. 5a, that the asymptote, and hence the onset of debond arrest, shifts left (i.e. towards lower conjugate bond zone sizes) as $\psi$ increases. Hence, for small enough initial debonds, arrest occurs sooner as the taper angle is increased for this range of values of $\psi$, while larger initial debonds become catastrophically unstable. However, for the highest taper angles $\left(\psi\right.$ larger than approximately $\left.80^{\circ}\right)$, it is seen from Fig. $5 \mathrm{~b}$ that the asymptote shifts right, towards higher values of $a^{*}$. That is, arrest occurs later and (stable) debonding occurs at lower load levels for taper angles above a critical level. Comparing these characteristics with those discussed in Bottega (1995), concerning the effects of varying the relative lengths and moduli of an untapered patch, it can be observed for the present case that when the taper angle is low, the patched structure assumes the characteristics attributed to shortening of the patch while, when the tapering becomes large enough, the overall stiffness of the composite structure is affected to the degree that the patched structure assumes the behavior associated with a more compliant patch. It may also be noted that the 'shifting' of the value of $a^{*}$ associated with the transition from full contact configurations to configurations with edge-point contact follows the same general trends as those associated with the shifting of the asymptote of the threshold curves as a function of the taper angle. We next consider the behavior of cylindrical structures. 
(a)

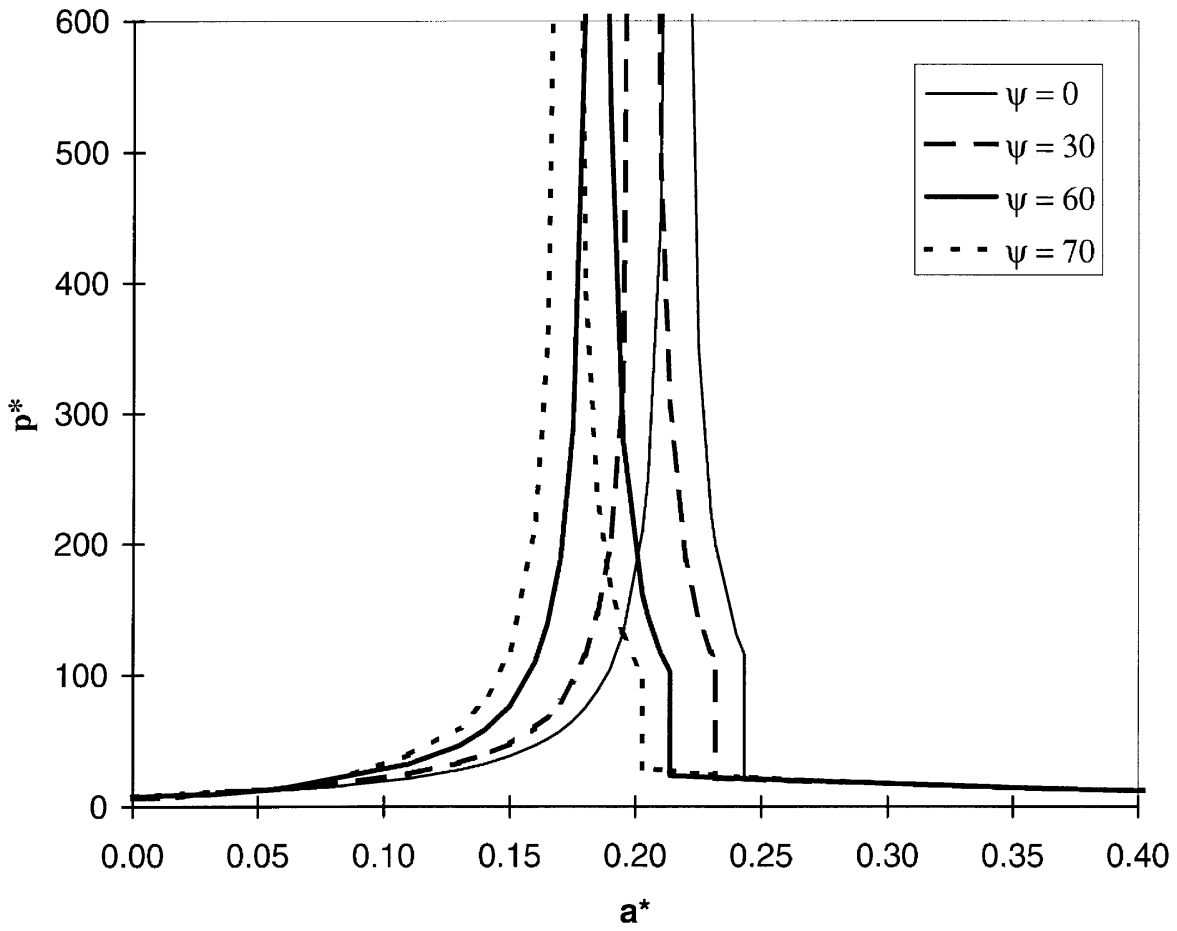

(b)

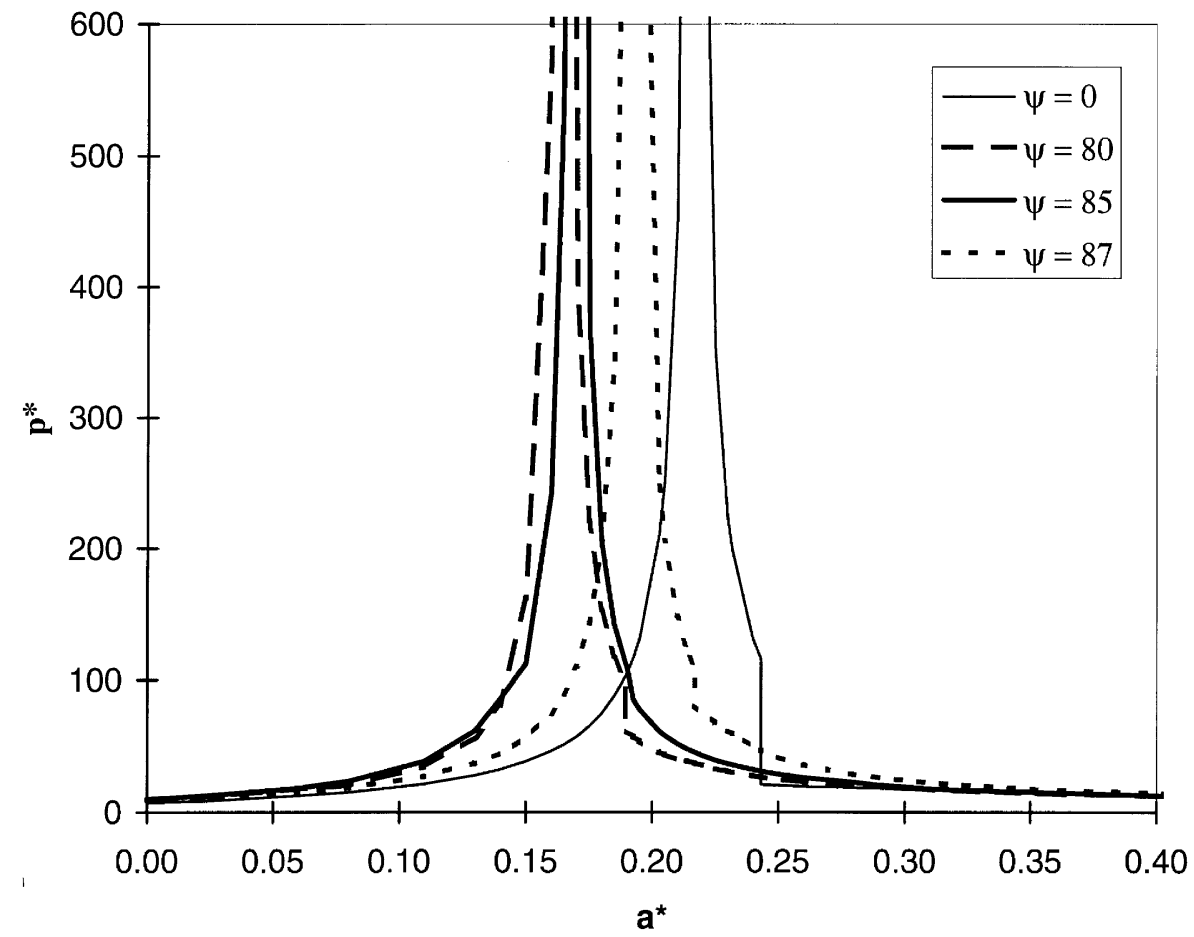

Fig. 5. Threshold curves/delamination paths corresponding to patched plates with clamped-fixed supports, for various taper angles: (a) renormed pressure vs conjugate bond zone size for $\psi=0,30,60$ and $70^{\circ}$; (b) renormed pressure vs conjugate bond zone size for $\psi=0,80,85$ and $87^{\circ}$. 


\section{Evolution of cylindrical structures}

In this section, results are presented for the case of patched cylindrical structures. As was done in the previous section for flat structures, we consider the two opposite extremes of support conditions: (i) hinged - so as to allow rotation and free-so as to allow in-plane translation (hinged-free), and (ii) clamped - so as to prohibit rotation and fixed-so as to prohibit in-plane translation (clamped-fixed). Cylindrical structures where the edges of the base panel have hingedfree supports will be considered first.

\subsection{Hinged-free supports}

We first consider a patched cylindrical structure where the edges of the base panel are free to rotate and are also free to translate circumferentially. We recall that in Part 1 of this study (Bottega and Karlsson, 1998) it was shown that the debonded region will not possess a contact zone for the case of hinged supports (free or fixed). Similar arguments also preclude the occurence of edge contact. We therefore consider propagation of the debonded region with the entire debonded segment of the patch lifted away from the base panel for the present support condition. The results for the present case are qualitatively and quantitatively similar to those for the flat panel with hinged-free supports presented in section 3.1. The discussion of the present results is therefore abbreviated.

Threshold curves expressed in terms of the renormed pressure $p^{*}$ as a function of the conjugate bond zone size $a^{*}$, and the renormed centerspan deflection $\Delta_{0}^{*}$ as a function of $a^{*}$, for taper angles $\psi=75,80$, and $85^{\circ}$ together with the path for the corresponding untapered patch, $\psi=0^{\circ}$ (Bottega and Loia, 1996), are displayed in Figs 6a and 6b, respectively. (The right vertical axes are rescaled, multiplied/divided by $L_{0}^{2}$, to allow for direct comparison with the corresponding results for flat structures-Figs $3 \mathrm{a}$ and $3 \mathrm{~b}$.) The corresponding stiffness degradation curves are displayed in Fig. $6 \mathrm{c}$ (with the right vertical axis rescaled accordingly to allow for direct comparison with Fig. 3c.) As for the analogous flat structure, the discontinuities (steps) in the threshold curves arise from the discontinuities (steps) in the structure at the points where a new layer comes into effect as $a^{*}$ is varied.

The qualitative features for both pressure controlled loading and deflection controlled loading are seen to directly correlate with those for the case of the flat plate. That is, the threshold curves for the pressure controlled case are monotonically decreasing, suggesting unstable and catastrophic debonding for this case. Likewise, the threshold curves for the deflection controlled case possess a minimum suggesting unstable and catastrophic debonding, unstable followed by stable debonding, or stable debonding depending on the initial size of the debonded region. It is also seen that for hinged-free support conditions the paths for the cylindrical structure and the flat structure are almost identical quantitatively as well. This may be attributed to the fact that for free support conditions, the membrane force vanishes eliminating the pressure-membrane coupling associated with nonvanishing initial curvature of the (shallow) shell. Thus these minimally constrained structures behave almost identically. This will not be so for the case of clamped-fixed supports presented in the next section, where nonvanishing initial curvature has a dramatic influence on the debonding behavior of the structure. 

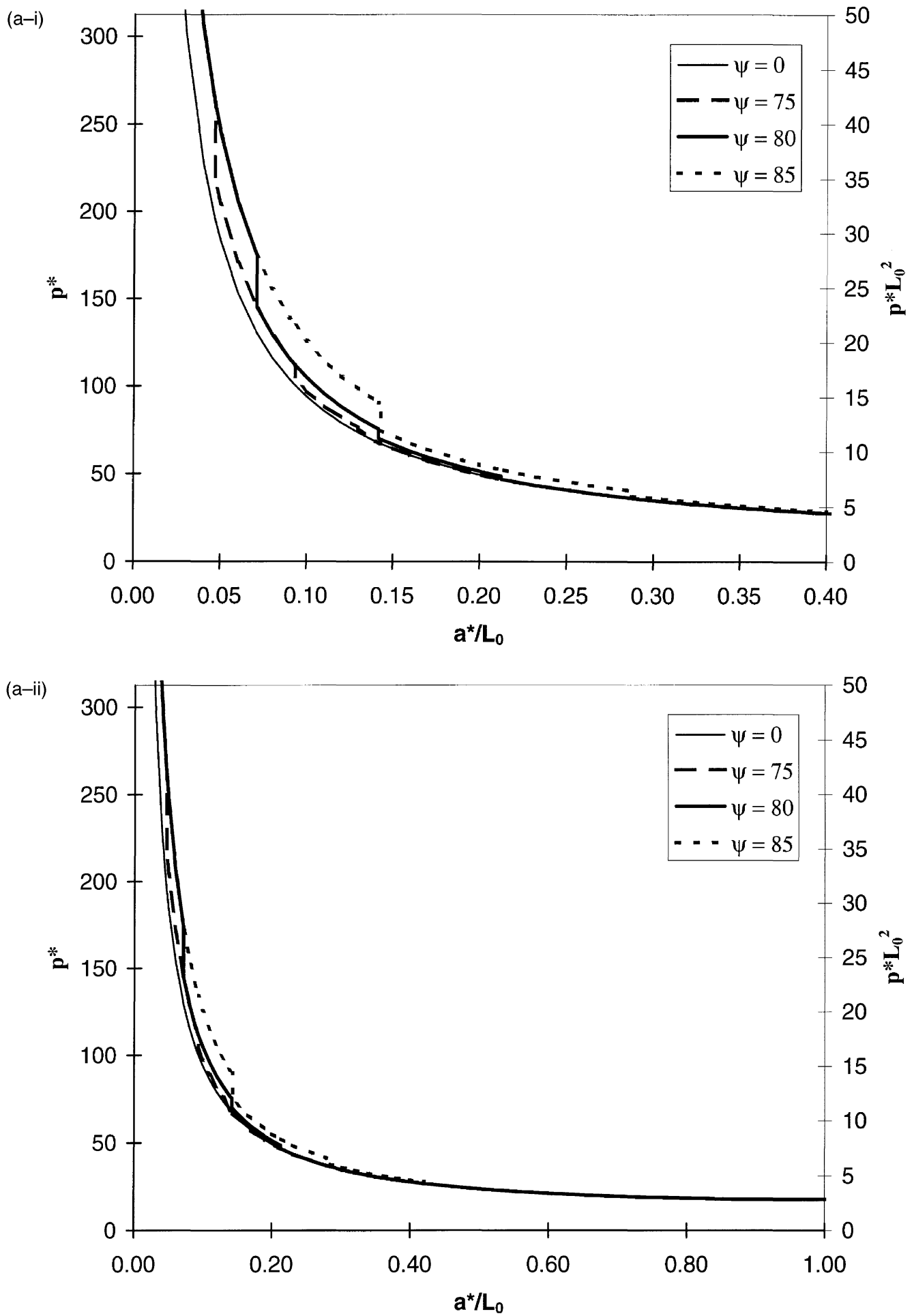

Fig. 6. Threshold curves/delamination paths corresponding to patched cylindrical panels with hinged-free supports, for various taper angles $\left(\psi=0,75,80\right.$ and $\left.85^{\circ}\right)$ : (a-i,ii) renormed pressure vs conjugate bond zone size; (b) renormed centerspan deflection vs conjugate bond zone size; (c) stiffness degradation curves. 
(b)

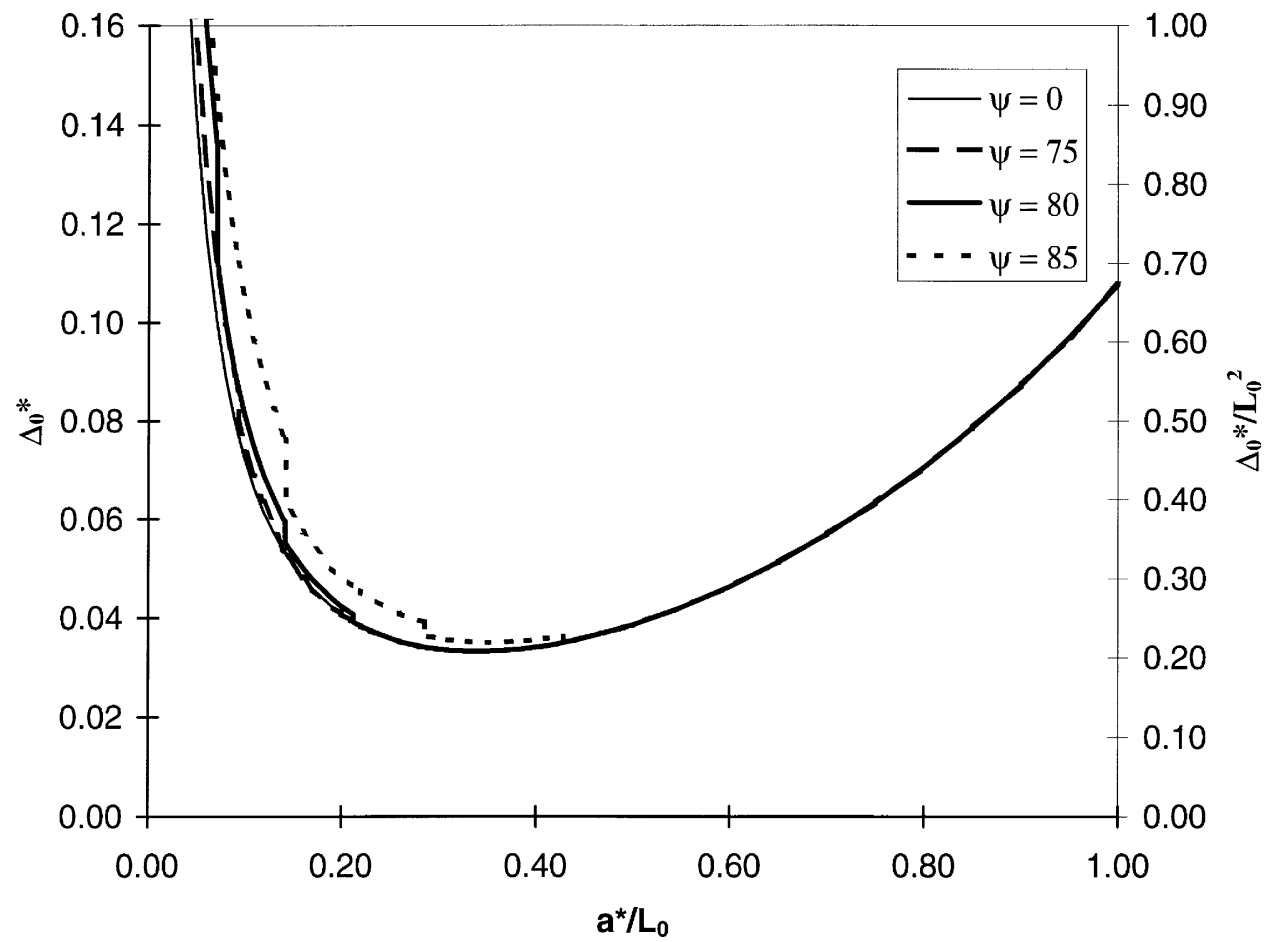

(c)

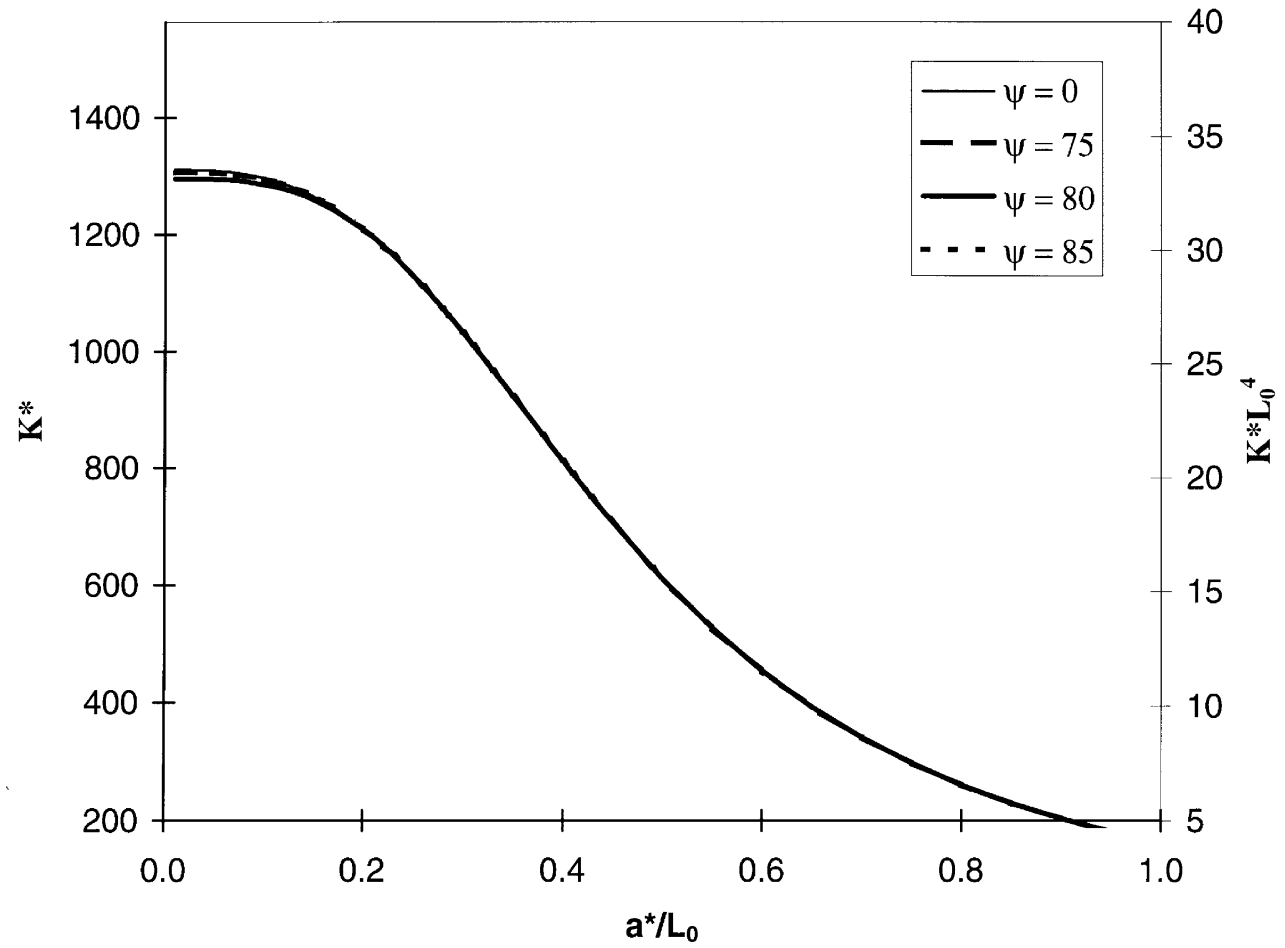

Fig. 6-continued. 


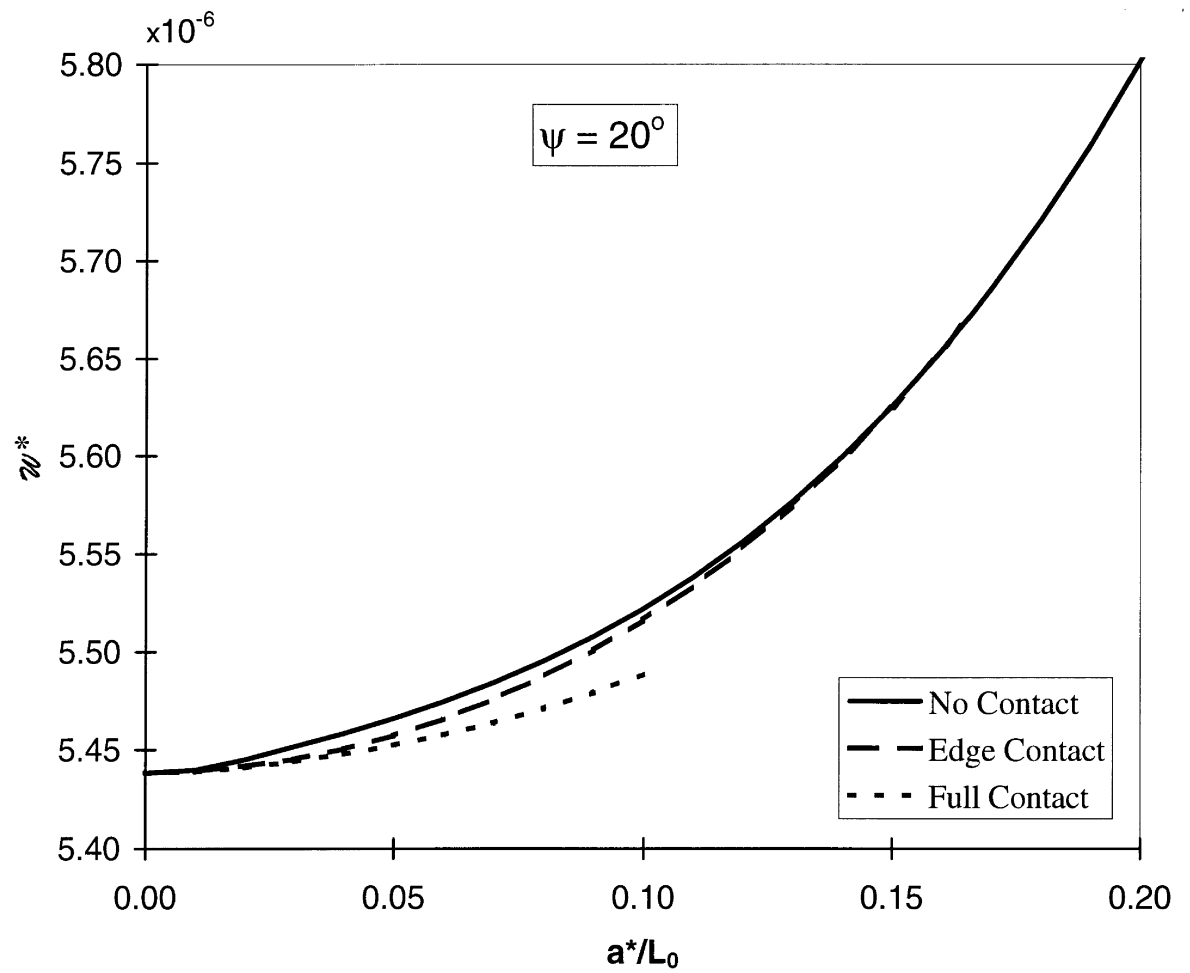

Fig. 7. Total normalized work per unit pressure vs conjugate bond zone size for a representative patched panel with clamped-fixed supports, $\psi=20^{\circ}$.

\subsection{Clamped-fixed supports}

We next consider the evolution of the composite shell structure when the edge supports are clamped - so as to prohibit rotation and fixed-so as to prohibit circumferential translation. For the present case we consider taper angles, $\psi$, in the range $10^{\circ} \leqslant \psi \leqslant 87^{\circ}$.

We first recall from Part 1 (Bottega and Karlsson, 1998), that for clamped support conditions (fixed or free), the configuration of the debonding structure for which a full contact zone is present, or that for which edge-point contact occurs, as well as the configuration in which no contact of the debonded segments occurs, are all possible. As for the corresponding case of a flat structure, in instances where all three configurations are admissible (physically realizable solutions can be found), the 'preferred' configuration will be taken to be the one corresponding to the lowest total energy of the system as characterized by the work per unit pressure detailed by eqn (6). The work per unit pressure $\mathscr{W}^{*}$, for the case of $\psi=20^{\circ}$, is displayed in Fig. 7. (We point out that the cut off for the edge contact curve occurs at $a^{*} / L_{0}=0.165$.) As for the corresponding flat structure, the hierarchy of admissible configurations is found to be: (i) full contact zone; (ii) edge-point contact; (iii) no contact. Similar results were obtained for all ranges of taper angles, but are omitted for brevity. Threshold curves, expressed in terms of the renormed pressure $p^{*}$ as a function of the conjugate bond zone boundary $a^{*}$ for the representative taper angles $\psi=20^{\circ}$ and $\psi=86^{\circ}$, are 
displayed in Figs 8a and 8b-i,ii, respectively, for each of the three possible configurations (where admissible). The corresponding threshold curves expressed in terms of the renormed centerspan deflection, and the associated stiffness degradation curves for the larger representative taper angle $\left(\psi=86^{\circ}\right)$ are displayed in Figs $8 \mathrm{c}$ and $8 \mathrm{~d}-\mathrm{i}$, ii, respectively. We note that, for any value of $\psi$, the paths associated with no contact approach the corresponding paths for the untapered case (Bottega and Loia, 1996) and become identical to those paths once the last step of the patch is encountered. As such, all paths will be identical to the no contact paths of Figs 8b-ii, 8c and 8d-ii for large enough values of $a^{*}$. Let us first consider the smaller taper angle $\left(\psi=20^{\circ}\right)$. It is seen from Fig. 8a that when the pressure level achieves that indicated for the preferred configuration for any given initial debond size, debonding ensues and progresses in an unstable and catastrophic fashion. It may also be seen that for small initial debond sizes $a^{*} / L_{0}=a_{0}^{*} / L_{0}<0.104$, debonding begins and progresses with a full contact zone present until $a^{*} / L_{0}=0.104$ at which time the debonded segment lifts away at all interior points of the debonded region, but maintains edge-point contact until $a^{*} / L_{0}=0.165$. At this instant complete 'lift off' occurs and unstable debonding continues catastrophically, with no contact of the debonded segments of the patch and base panel occuring. For initial conjugate bond zone sizes within the range $0.104<a_{0}^{*} / L_{0}<0.165$, debonding occurs when the threshold level indicated by the edge contact path is achieved and progresses in an unstable manner with edge-point contact until $a^{*} / L_{0}=0.165$ at which time 'lift off' occurs and unstable and catastrophic debonding proceeds with no contact. (It may be noted that the critical pressure in this range of $a^{*} / L_{0}$ is lower than would be predicted if edge-point contact were neglected.) For $a_{0}^{*} / L_{0}>0.165$ unstable and catastrophic, unstable followed by stable, or stable debonding ensues without contact, depending on the initial conjugate bond zone size, when the indicated critical pressure is achieved. The debonding scenario for the patched panel with the larger taper angle $\left(\psi=86^{\circ}\right)$ is quite different. It is seen from Fig. $8 \mathrm{~b}$ that the threshold curve (of the 'preferred' full contact configuration) possesses an asymptote at $a^{*} / L_{0}=0.023$, indicating that structures possessing initial debonds such that $a_{0}^{*} / L_{0}<0.023$ grow in a stable manner once debonding ensues with growth effectively arresting as the asymptote is approached. In contrast, initial debonds such that $a_{0}^{*} / L_{0}>0.023$ will propagate in an unstable and catastrophic, an unstable followed by stable, or in a stable manner once the critical pressure is reached, progressing from configurations of full contact to edge-point contact at $a^{*} / L_{0}=0.113$, to no contact at $a^{*} / L_{0}=0.205$, depending on the initial location of the conjugate bond zone boundary as indicated. With the debonding scenarios for the above two representative taper angles established, we next examine the effects of varying the degree of tapering.

Threshold curves corresponding to the taper angles $\psi=10,20$, and $40^{\circ}$ are displayed in Fig. 9a, those corresponding to $\psi=60,80$, and $82^{\circ}$ are displayed in Fig. $9 \mathrm{~b}$, and the paths for $\psi=84,86$, and $87^{\circ}$ are shown in Fig. $9 \mathrm{c}$. In each figure, the threshold curve corresponding to the untapered case $\left(\psi=0^{\circ}\right)$ is also exhibited for comparison. For each taper angle, the path segment corresponding to the 'preferred' configuration is shown for the associated range of $a^{*}$, with the right most dis-

\footnotetext{
$\$$ While the transition from full contact to edge-point contact configurations (which is associated with the passing of the inflection point from one side of the propagating bond zone boundary to the other, as $a^{*}$ increases) is indicated by a discontinuity in the corresponding threshold curve, the transition from edge-point contact to no contact is apparently smooth.
} 
(a)
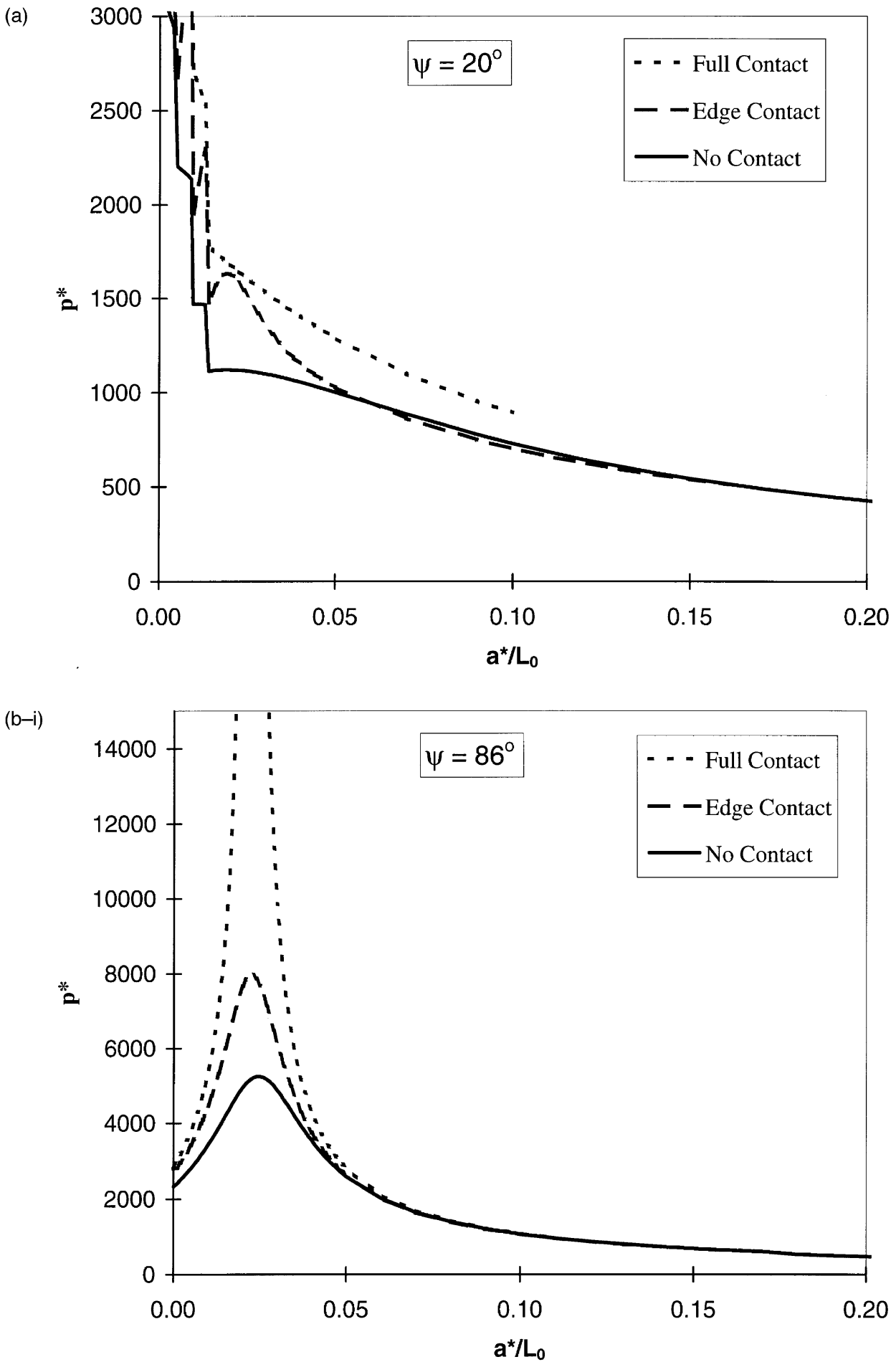

Fig. 8. Comparison of threshold curves corresponding to configurations with full contact zone, edge-point contact and no contact, for representative patched panels with clamped-fixed supports. (a) renormed pressure vs conjugate bond zone size for $\psi=20^{\circ}$; (b-i,ii) renormed pressure vs conjugate bond zone size for $\psi=86^{\circ}$; (c) renormed centerspan deflection vs conjugate bond zone size for $\psi=86^{\circ}$; (d-i, ii) stiffness degradation curves for $\psi=86^{\circ}$. 


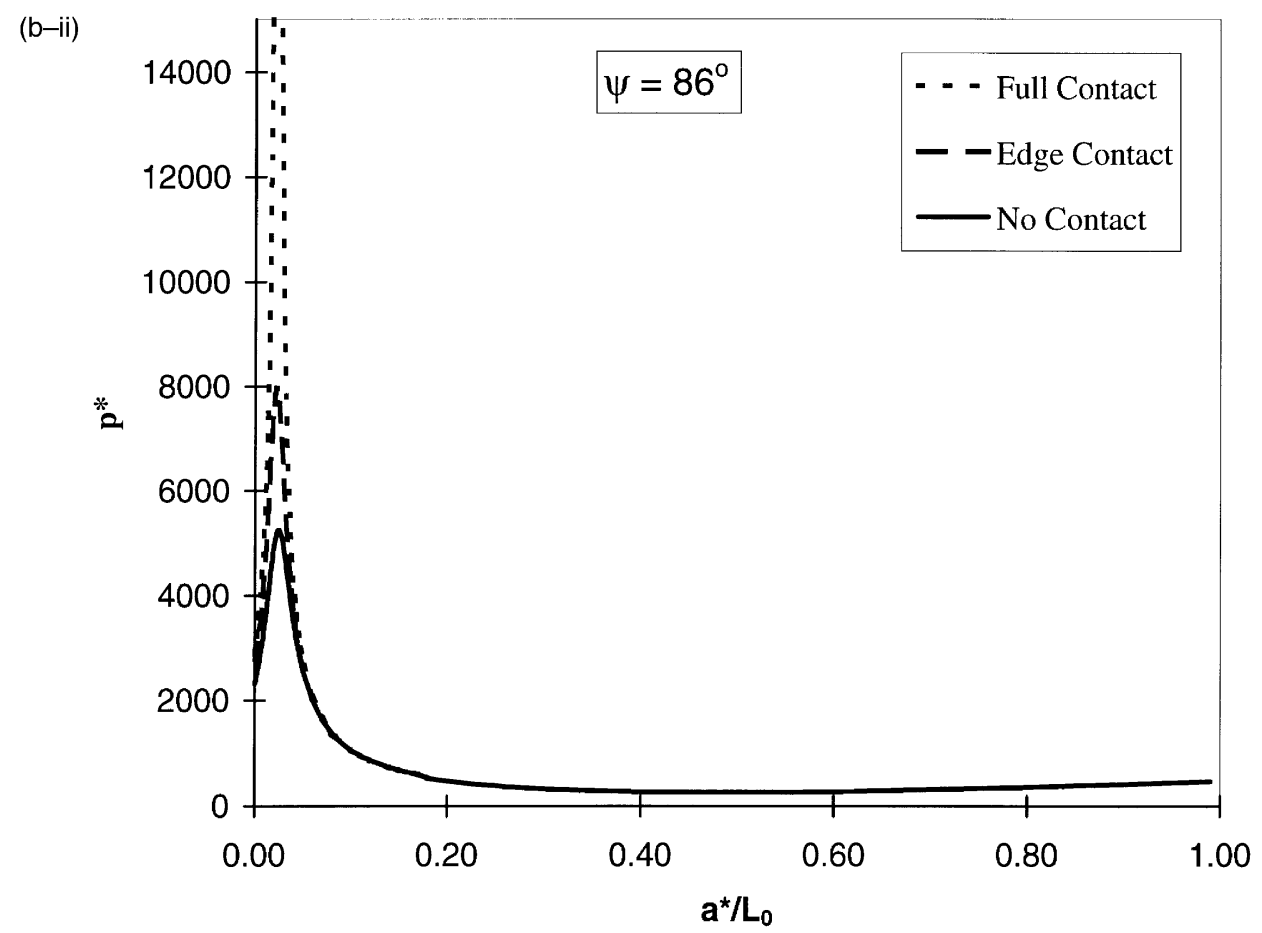

(c)

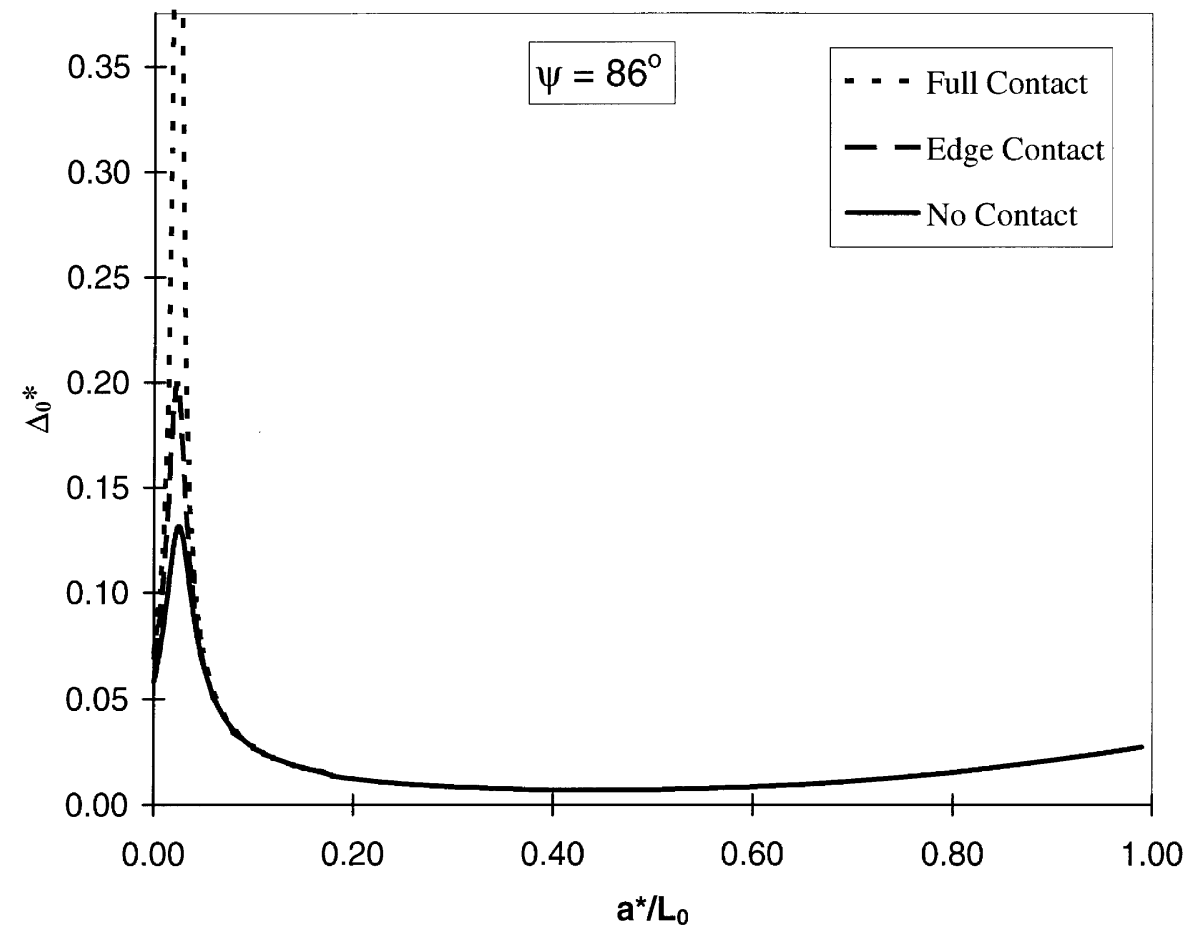

Fig. 8-continued. 

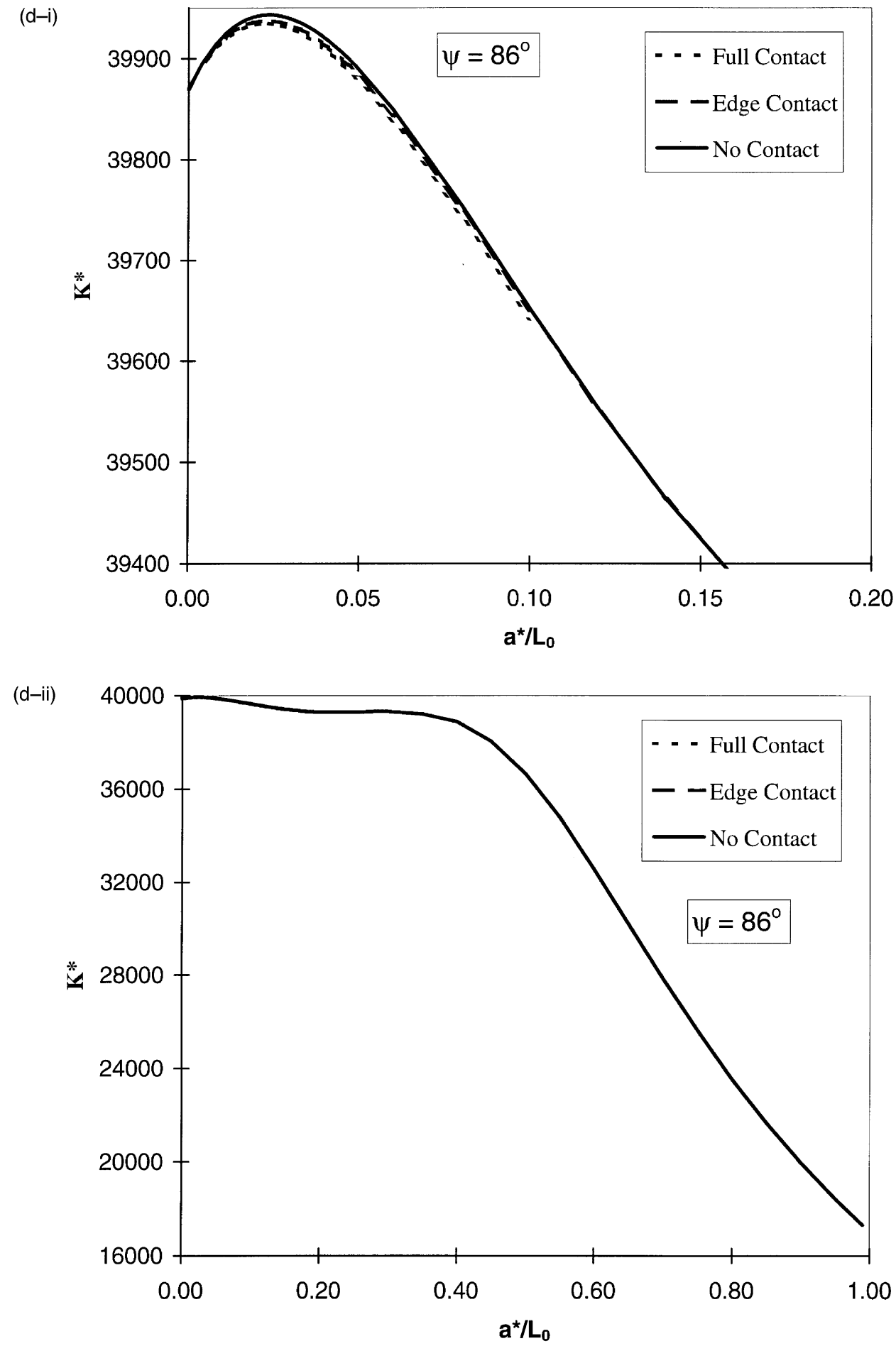

Fig. 8-continued. 
(a)

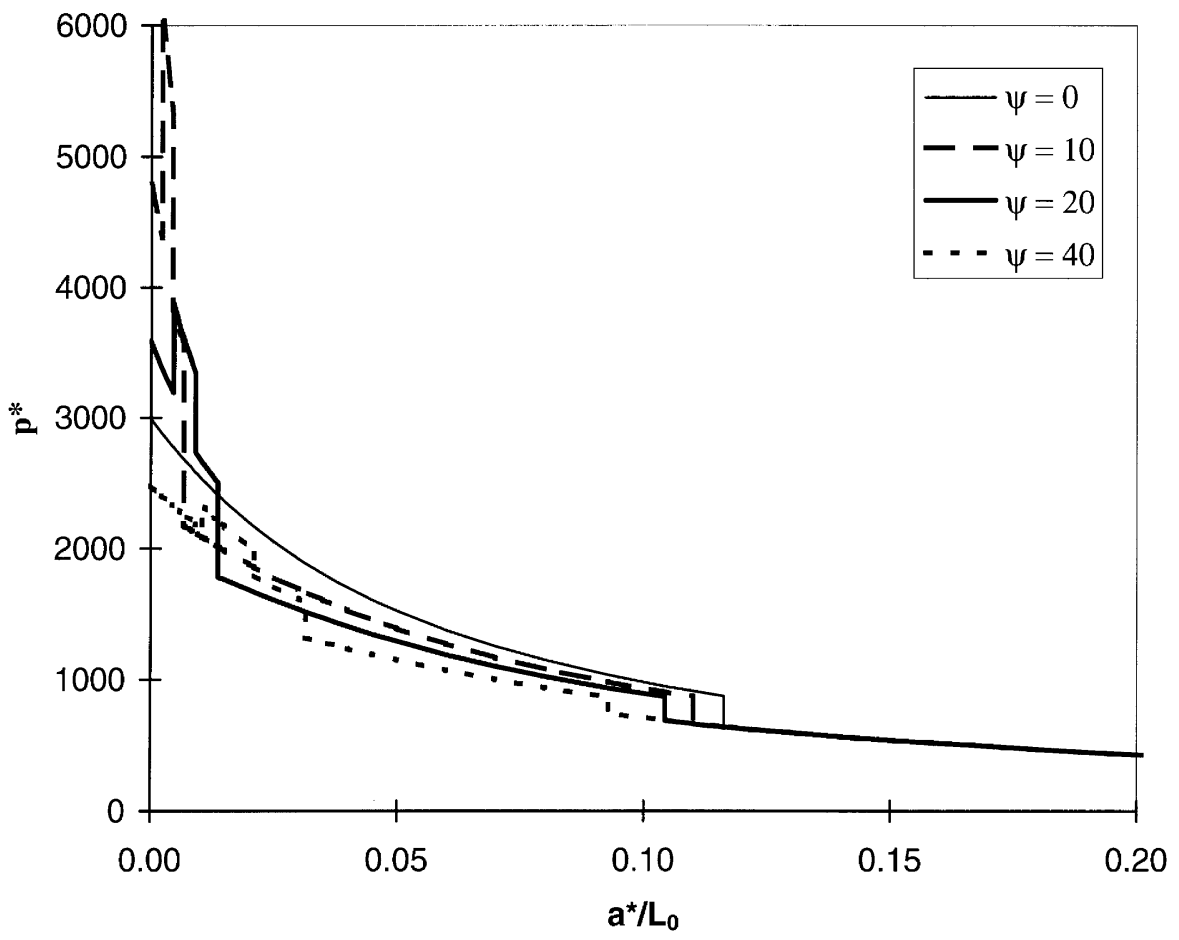

(b)

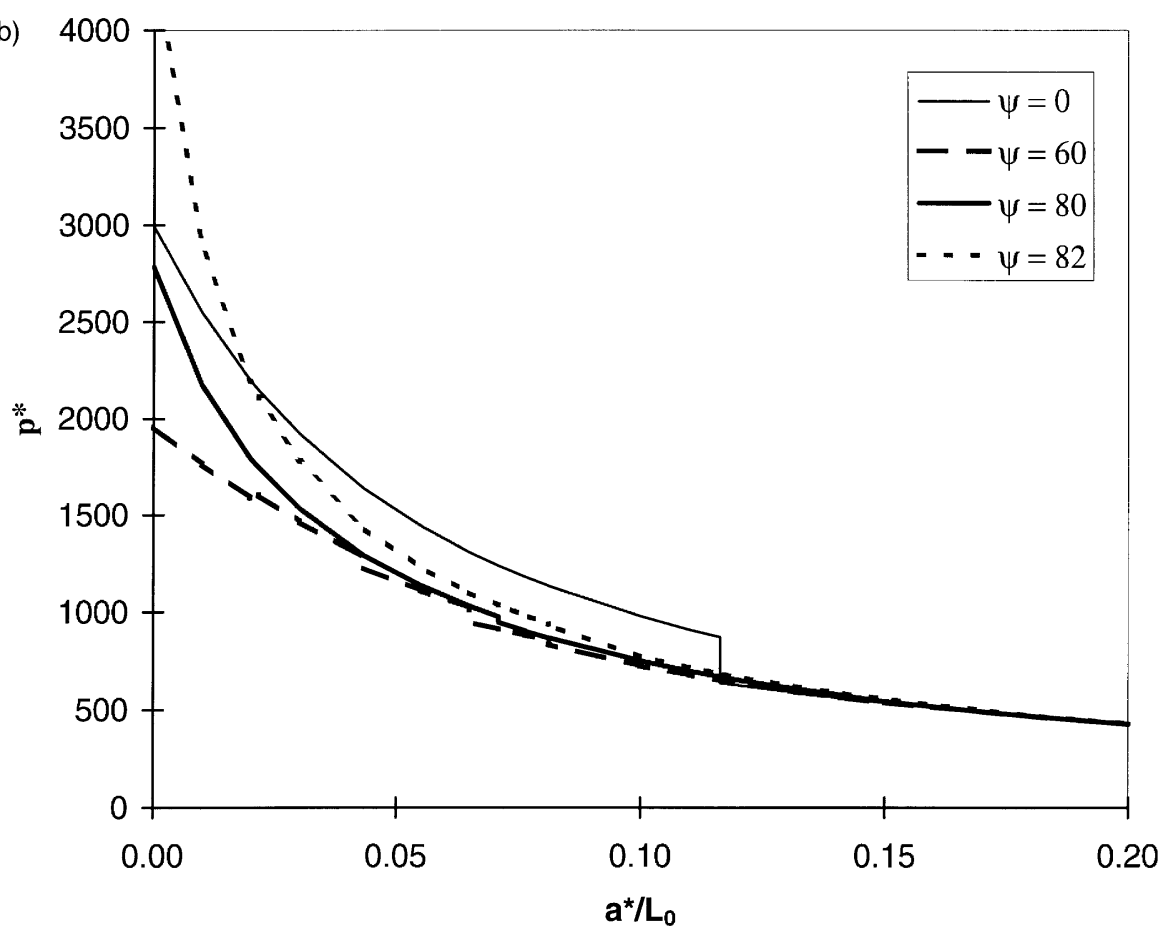

Fig. 9. Threshold curves/delamination paths corresponding to patched cylindrical panels with clamped-fixed supports, for various taper angles: (a) renormed pressure vs conjugate bond zone size for taper angles $\psi=0,10,20$ and $40^{\circ}$; (b) renormed pressure vs conjugate bond zone size for taper angles $\psi=0,60,80$ and $82^{\circ}$; (c) renormed pressure vs conjugate bond zone size for taper angles $\psi=0,84,86$ and $87^{\circ}$. 


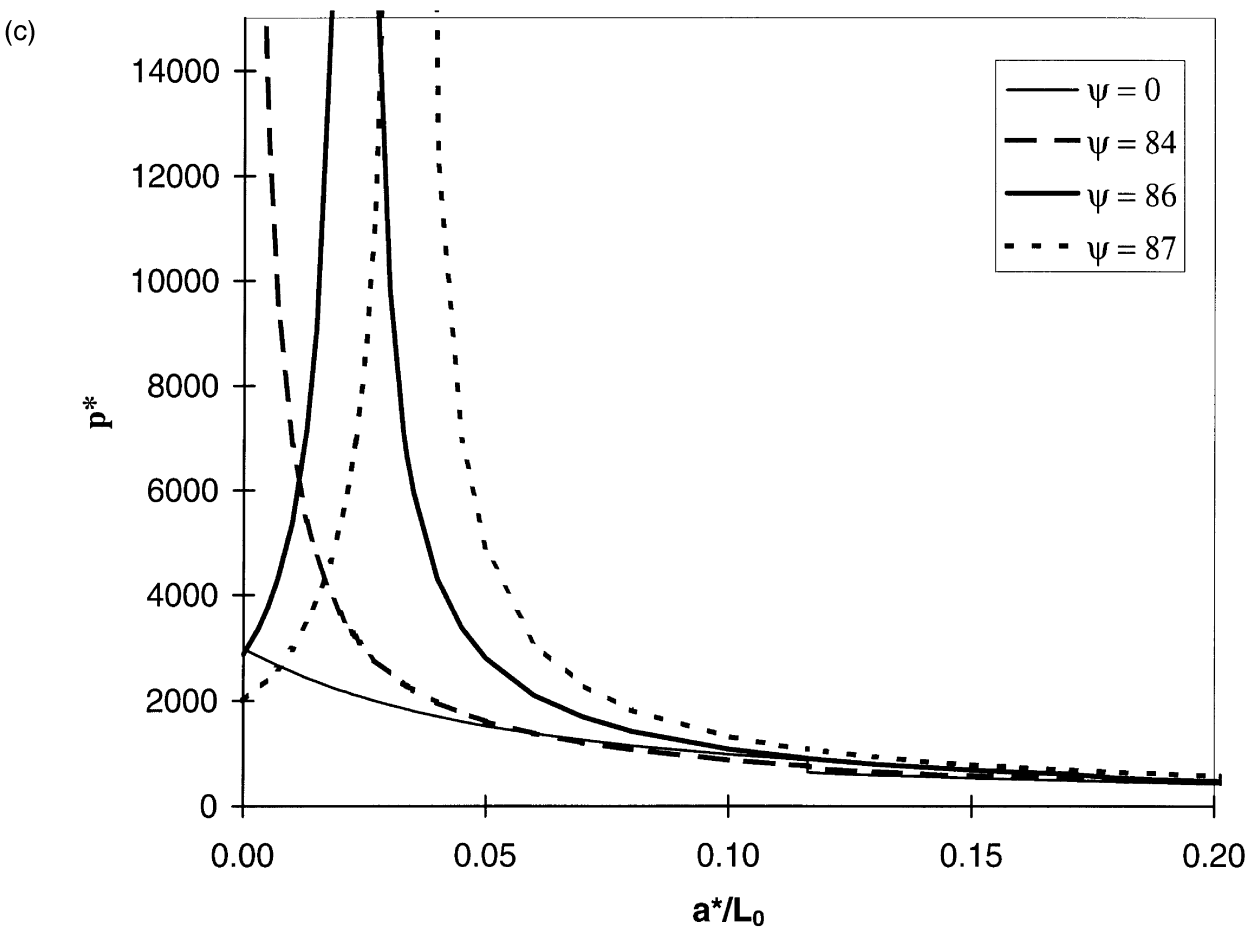

Fig. 9-continued.

continuity for that path generally corresponding to the transition from full contact configurations to edge contact configurations. The magnitude of the jump in the threshold curves associated with this transition is seen to diminish as the taper angle increases. Moreover, for the highest taper angles considered, $\psi=84,86$ and $87^{\circ}$, the respective transitions occurring at $a^{*} / L_{0}=0.092,0.113$, and 0.131 , are imperceptible within the resolution of the figure. The remaining discontinuities, most visible in Fig. 9a, occur at the locations of the steps for each particular patch. It is seen that the threshold curves corresponding to the two highest taper angles, $\psi=86^{\circ}$ and $\psi=87^{\circ}$, possess asymptotes separating regions of stable and unstable debonding as detailed earlier. The asymptotes are seen to shift left as the taper angle diminishes, ultimately shifting out of the range of physically meaningful values of $a^{*}$ for $\psi \leqslant 84^{\circ}$. Thus the threshold curves for the majority of taper angles are seen to possess only an 'unstable' branch, for the specific structures under consideration. It is also seen that the threshold curves corresponding to the moderately large taper angles $\psi=40,60$ and $80^{\circ}$, lie below the curve corresponding to the untapered patch for the entire range of $a^{*}$. Thus, tapering within this range of taper angles actually decreases the effectiveness of the patch, with the onset of unstable and catastrophic debonding occuring at lower pressure levels than is indicated for the untapered patch. This situation is alleviated somewhat for patches which are initially fully bonded $\left(a_{0}^{*} / L_{0}=0\right)$ or almost fully bonded for the taper angles $\psi=82^{\circ}$ and $\psi=84^{\circ}$, though only for a small range of conjugate bond zone sizes. Similar behavior is indicated for the low taper angles considered, $\psi=10^{\circ}$ and $\psi=20^{\circ}$, following very limited unstable debonding, intermittent arrest (when the propagating bond zone boundary encounters the first step) followed by cata- 
strophic debonding is indicated for patches which are initially fully or very nearly fully bonded to the base structure. It is thus seen that slight tapering or severe tapering increases the initial threshold pressure for patches with effectively no initial debonding at their edges, with only the most severe tapering seen to stabilize the debonding process.§ Degrees of tapering corresponding to taper angles outside these narrow bounding ranges of taper angle are seen to actually worsen conditions with regard to debonding as compared to having no tapering of the patch edge at all.

\subsection{Comments on the influence of delamination modes for cylindrical structures}

With regard to the influence of delamination modes, we consider the possibility that the debonding scenarios discussed may be altered somewhat if the delamination mode ratio varies radically and in a suitable fashion, as the bond zone boundary propagates under interface conditions in which 'mode mixity' (see, for example, Hutchinson and Suo, 1992) is an issue. This is found not to be the case, however, for the loading case considered. Calculations of the 'large scale' patchbase panel interfacial stresses [see Appendix A of Part 1 (Bottega and Karlsson, 1998)] at the bond zone boundary show that, in general, the corresponding normal stress $\sigma^{(1)} 1\left(a^{*}\right)$ is negative and the associated shear stress $\tau^{(1)} 1\left(a^{*}\right)$ maintains the same sign for all $a^{*}$. For the highest taper angles (clamped supports), $\sigma^{(1)} 1\left(a^{*}\right)$ may be positive for very small conjugate bond zone sizes $\left(a^{*} / L_{0}<0.015\right)$, but the magnitude of the normal stress is always much smaller than that of the corresponding shear stress $(<1 \%)$ for these cases. (In such isolated and restricted situations, the shear to normal stress ratio increases as $a^{*}$ proceeds away from the edge toward the location of the asymptote within the range of values of the conjugate bond zone boundary corresponding to the stable branch of the associated threshold curve. Any variation in fracture toughness may thus enhance the predicted stable behavior, but the overall qualitative behavior would be unaltered). In view of the above, if $\sigma_{1}^{(1)}$ and $\tau_{1}^{(1)}$ are considered to represent the 'distant loading' (or some average measure) with regard to local small scale effects, then their characteristics appear to support the notion that the local ('small scale') stress field, and hence the local mode ratio/phase, generally maintains pure mode-II for the cases considered. If this is so, and in view of the above, it is anticipated that the delamination modes have little influence on the debonding scenarios discussed for the loading condition considered.

\section{Concluding remarks}

In this the second part of a two part study, the problem of edge debonding of step-tapered patches from both flat and cylindrical panels subjected to pressure loading has been considered.

\footnotetext{
$\S$ Consideration of the range of results for untapered patches on cylindrical structures presented in (Bottega and Loia, 1996) suggests that the effect of introducing a small degree of taper is similar to that of shortening an untapered patchthe threshold curves shifted leftward/downward with increasing $\psi$, while the effect of introducing a severe taper is similar to that of decreasing the stiffness of an untapered patch (the threshold curves shifted to the right/upward with increasing $\psi)$.

- It is interesting to note that relatively compliant patches (those of low modulus ratio, as is the case for bonded sensors for example) exhibit an even richer assortment of behaviors. Corresponding results are presented as an addendum in the appendix.
} 
Numerical simulations based on analytical solutions of the linearizion of the general formulation presented in Part 1 of this study (Bottega and Karlsson, 1998) were performed, and results based on these simulations presented. The effects of support conditions were also examined. Configurations corresponding to full contact of the debonded segment of the patch with the base plate, contact of the edge of the debonded segment alone, and no contact of the debonded segments were included in the formulation and each were found to occur at various stages in the evolution of the debonding structures, depending on the support conditions and the degree of taper. It was seen that failure to include the effects of contact can drastically change the predicted critical load levels and the corresponding behavior of the evolving composite structure. Though tapering is often introduced in practice for the purpose of reducing 'edge effects' and thus may be thought to improve the resistance of the structure to edge debonding, it was observed that while this goal is sometimes achieved, the taper angle and the manner of support affect the debonding behavior of the composite structure in a complex and often unanticipated manner. In particular, it was seen that the introduction of edge taper often lowers the critical load level below that which is predicted for a corresponding untapered patch, often initiating unstable and catastrophic debonding for a wide range of taper angles and thus inducing the opposite effect of that for which it was intended. It is thus seen that edge-tapering of doublers may often have detrimental rather than positive effects with regard to debonding, from which it may be concluded that such modifications of the patch should be carefully considered.

\section{Acknowledgements}

The authors wish to thank D.W. Oplinger of the Federal Aviation Administration for his support and encouragement. This work was supported by the FAA through the Rutgers University Center for Computational Modeling of Aircraft Structures (CMAS).

\section{Appendix: addendum}

For the reasons given at the end of section 2, the results presented throughout have been for doublers whose stiffnesses in the untapered region of the patch match those of the base structure, that is for patches of modulus ratio $E_{\mathrm{p}}=1.0$, with total thickness the same as that of the base structure. While the overall effects of reducing (or enhancing) the stiffness of the (untapered) patch were considered in prior studies (Bottega, 1995; Bottega and Loia, 1996) for corresponding structures, the influence of edge taper on the behavior of relatively compliant patches shows some interesting behavior not seen in the results for patches of unit modulus ratio. In this regard, threshold curves corresponding to a cylindrical panel for the case where the modulus of a patch is one tenth that of the base structure, $E_{\mathrm{p}}=0.1$, are displayed in Figs A1a and A1b. While, as for the untapered structures of the prior studies, the threshold curves are seen to possess an asymptote(s) separating a stable and unstable branch(es), the presence of the taper is seen to introduce alternate regions of stable and (bounded) unstable debonding and to shift the asymptotes left. The latter indicates that the large scale stability of debonding for small initial debonds is enhanced (effective arrest occurs sooner), and the former indicates that the corresponding small scale stability is 
(a)

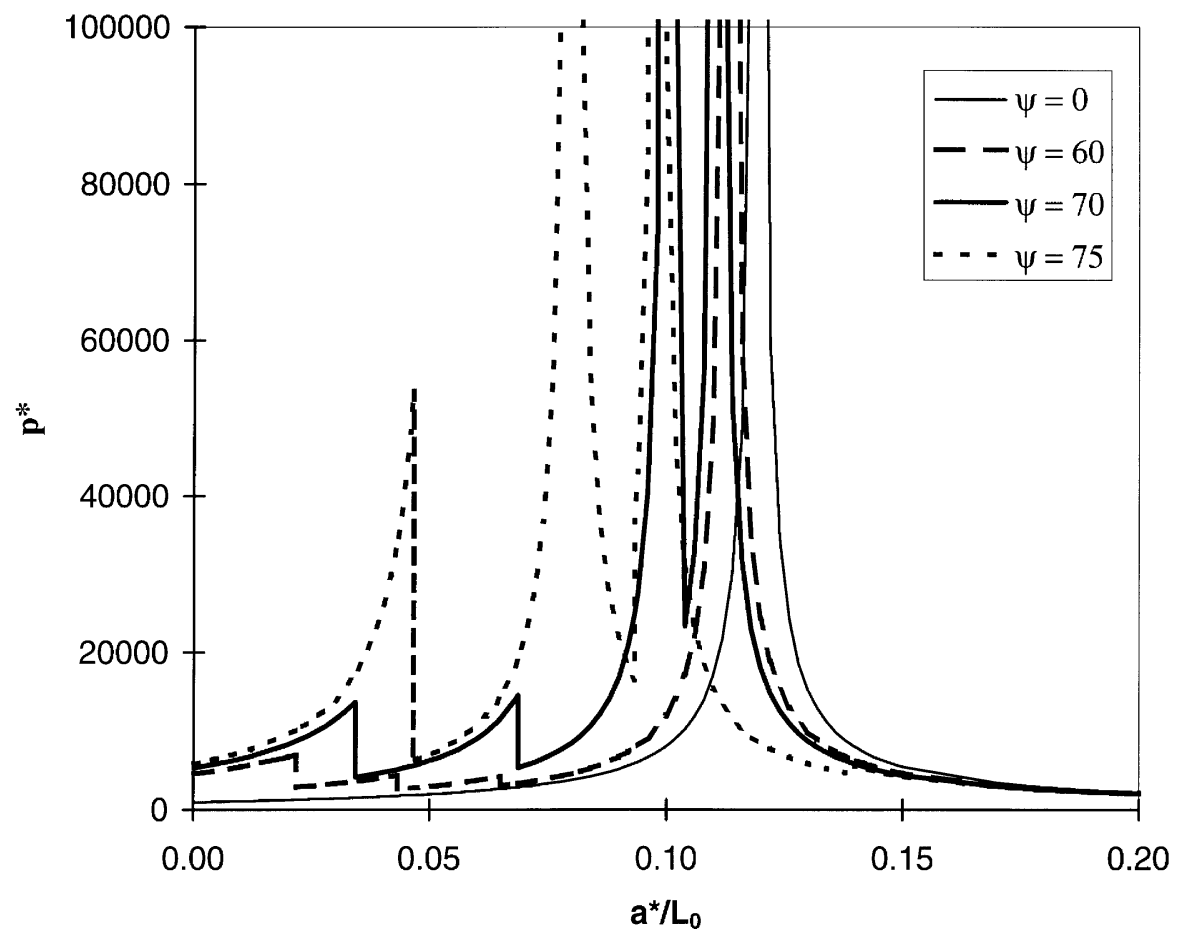

(b)

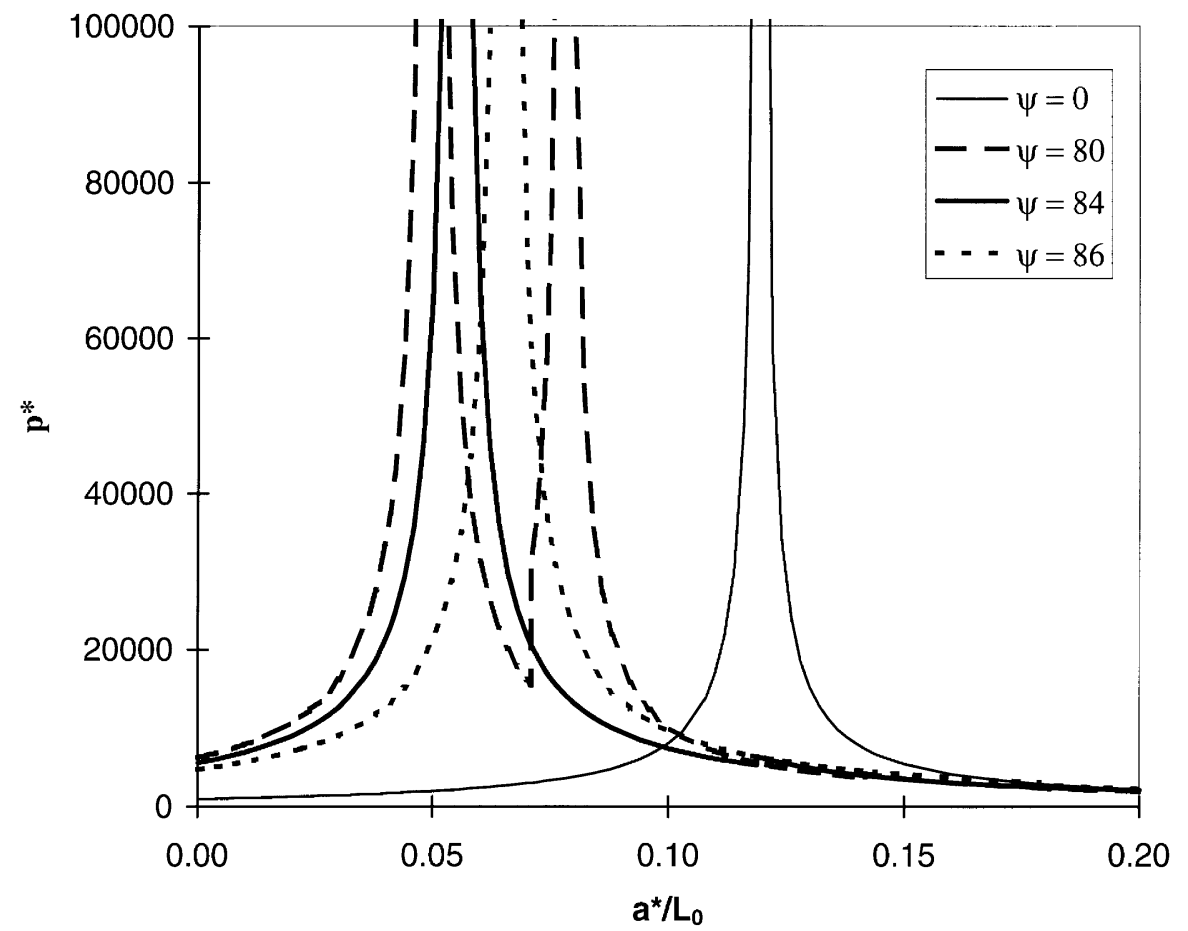

Fig. A1. Threshold curves/delamination paths corresponding to cylindrical panels with 'compliant patches' $\left(E_{\mathrm{p}}=0.1\right)$ and clamped-fixed supports, for various taper angles: (a) renormed pressure vs conjugate bond zone size for $\psi=0,60$, 70 and $75^{\circ}$; (b) renormed pressure vs conjugate bond zone size for $\psi=0,80,84$ and $86^{\circ}$. 
diminished for small initial debonds associated with moderately tapered patches. The threshold level for the onset of debonding for the compliant patch is seen to be elevated by the introduction of edge taper for all cases shown.

\section{References}

Bottega, W.J., 1995. Separation failure in a class of bonded plates. Composite Structures 30 (3), 253-269.

Bottega, W.J., Loia, M.A., 1996. Edge debonding in patched cylindrical panels. International Journal of Solids and Structures 33 (5), 3755-3777.

Bottega, W.J., Loia, M.A., 1997. Axisymmetric edge debonding in patched plates. International Journal of Solids and Structures 34 (18), 2255-2289.

Bottega, W.J., Karlsson, A.M., 1998. On the detachment of step-tapered doublers: Part 1-foundations. International Journal of Solids and Structures 36 (11), 1597-1623.

Hutchinson, J.W., Suo, Z., 1992. Mixed Mode Cracking in Layered Materials. In: J.W. Hutchinson Wu, T.Y. (Eds.), Advances in Applied Mechanics, 29. Academic Press, San Diego, pp. 63-191.

Loia, M.A., Bottega, W.J., 1995. On planar-tensile representation of edge debonding in patched panels under pressure. International Journal of Adhesion and Adhesives 15 (4), 211-217.

Raizenne, M.D., Heath, J.B.R., Gaudertt, P.C., 1995. Failure Analysis of Bonded Boron Doublers. Proceedings of the National Research Council of Canada Symposium on Composite Repair of Aircraft Structures, University of British Columbia, Vancouver, Aug. 9-10, pp. 10.1-10.30. 